Fiber photometry of maternal oxytocin pulses by Yukinaga et al.

\title{
Recording and neural circuit-based manipulation of the maternal oxytocin pulses in mice
}

\author{
Hiroko Yukinaga ${ }^{1}$, Mitsue Hagihara ${ }^{1}$, Kazuko Tsujimoto ${ }^{1}$, Hsiao-Ling Chiang ${ }^{1}$, \\ Shigeki Kato ${ }^{2}$, Kazuto Kobayashi ${ }^{2}$, and Kazunari Miyamichi ${ }^{1,3 *}$ \\ ${ }^{1}$ Laboratory for Comparative Connectomics, Riken Center for Biosystems Dynamics \\ Research, Kobe, Hyogo 650-0047, Japan \\ 2 Department of Molecular Genetics, Institute of Biomedical Sciences, Fukushima \\ Medical University School of Medicine, Fukushima 960-1295, Japan \\ ${ }^{3}$ PRESTO, Japan Science and Technology Agency, Kawaguchi, Saitama 332-0012, Japan \\ *Correspondence: kazunari.miyamichi@,riken.jp
}

\section{Summary}

For mammals, successful parturition and breastfeeding are critical to the survival of offspring. Pulsatile release of the hormone oxytocin mediates uterine contraction during parturition and milk ejection during lactation. These oxytocin pulses are generated by unique activity patterns of the central neuroendocrine oxytocin neurons located in the paraventricular and supraoptic hypothalamus. However, the maternal activities of oxytocin neurons remain elusive because most classical electrophysiological studies in anesthetized rats have lacked the genetically defined cell identity of oxytocin neurons. We herein introduce viral genetic approaches in mice to characterize the maternal pulsatile activities of oxytocin neurons by fiber-photometry-based chronic in vivo $\mathrm{Ca}^{2+}$ imaging. We also demonstrate the pharmaco-genetic manipulation of oxytocin pulses during lactation via activating a prominent pre-synaptic structure of oxytocin neurons defined by retrograde trans-synaptic tracing. Collectively, our study opens a new avenue for the neuroscience of maternal neuroendocrine functions.

\section{Introduction}

Oxytocin (OT) is a nine-amino-acid peptide hormone known for mediating uterine contraction during parturition and milk ejection during lactation ${ }^{1-3}$. This hormone is mostly derived from the OT neurons located in the paraventricular hypothalamus (PVH) and supraoptic hypothalamus and secreted into the blood circulation via axonal release from the posterior pituitary. OT and OT receptor (OTR) knockout postpartum mother mice disable the milk ejection reflex $^{4-6}$, that is, the active transfer of milk from alveolar storage to mammary ducts in response to a transient increase in circulating plasma OT. These observations demonstrate that OT is indispensable for breastfeeding in mice. By 
Fiber photometry of maternal oxytocin pulses by Yukinaga et al.

contrast, OT function in parturition is less apparent as mutant female mice exhibit no major abnormalities in parturition ${ }^{4-6}$. Nevertheless, a study by Douglas et al. ${ }^{7}$ suggested that OT neurons are active during parturition, OT antagonist slows parturition, and stressinduced delayed parturition can be rescued by OT administration. Therefore, OT, the most potent uterotonic agent in human and domestic animals for over 50 years ${ }^{8}$, also facilitates parturition in mice, although other redundant mechanisms can compensate for its absence.

In normal situations, OT neurons have a range of spontaneous firing patterns that maintain a low level of OT tone. During parturition and lactation, however, OT neurons are thought to fire more synchronously in bursts, resulting in pulsatile OT secretion, which is necessary for contraction of the uterine and mammary glands. Classical studies have characterized maternal OT neural activities most intensively by in vivo extracellular recording techniques in rats and rabbits under anesthesia ${ }^{1,9-12}$ or awake ${ }^{13-15}$. However, due to technical limitations, the identity of OT neurons in these studies was speculative, mostly based on their electrophysiological characteristics and axonal projection to the posterior pituitary, not on $O T$ gene expression. Previous studies were also limited by the small number of units able to be analyzed in each experiment in which no long-term chronic recording was possible. To pinpoint OT neural activities among many other hypothalamic neural cell types that project to the pituitary ${ }^{16,17}$ and make better use of the modern cell-type-specific tool kits for the manipulation of gene functions and neural activities $^{18}$, a mouse model for studies of parturition and lactation needs to be developed.

The neural circuit mechanisms by which OT modulates various social behaviors in mice have been receiving increasing attention in recent years, and cell-type-specific genetic and activity manipulation of the OT system has been reported in such contexts ${ }^{3}$. For instance, to monitor the activities of genetically defined OT neurons, Cre-dependent targeting of genetically-encoded $\mathrm{Ca}^{2+}$ indicators ${ }^{19,20}$ or ChR2-assisted cell type identification in electrophysiological recordings (Opto-Tag) ${ }^{21,22}$ were utilized in recent papers or preprints in studies on social coding or socio-sensory input to the OT neurons. These suites of studies exemplify how cell-type-specific recording tools in mice clarify our understanding of OT systems in the modulation of social and parental behaviors; however, classical maternal functions of OT remain uncharacterized, which motivated us to introduce in vivo $\mathrm{Ca}^{2+}$ imaging to illuminate maternal OT pulsatile activities during parturition and lactation.

How are OT neural activities shaped by afferent neural circuitry? Our current understanding is mostly based on classical non-cell-type specific lesion or electric stimulation data in postpartum rats during lactation ${ }^{23-25}$. Previous studies have suggested putative routes to transmit the sensory input from the nipples to the OT neurons, in 
Fiber photometry of maternal oxytocin pulses by Yukinaga et al.

addition to the potential modulatory functions of limbic structures. However, the direct pre-synaptic partners of OT neurons have remained uncharacterized, particularly in terms of maternal OT functions, which has hampered our understanding of how the OT system operates in the context of specific neural connectivity.

In the present study, we first aim to introduce fiber photometry ${ }^{26}$ to genetically defined OT neurons to characterize the temporal dynamics of OT neural activities in parturient and lactating female mice. We then apply Cre-dependent trans-synaptic tracing ${ }^{27}$ to map quantitatively the input to OT neurons in postpartum mother mice, with reference to nonpregnant females. Based on this map, we provide a proof-of-principle demonstration of pharmaco-genetic manipulations of a defined pre-synaptic structure to modulate maternal pulsatile OT neural activities. Collectively, these data form the basis to introduce modern neuroscience techniques in mice ${ }^{18}$ to the field of maternal neuroendocrine functions.

\section{Results}

\section{Activities of OT neurons during parturition}

To monitor selectively the activity dynamics of genetically-defined OT neurons in freemoving female mice, we first crossed OT-Cre mice ${ }^{28}$ with the Cre-dependent GCaMP6s driver line, Ai162 (ref. 29). As a target of fiber photometry recording ${ }^{26}$, we focused on the $\mathrm{PVH}$ because it contains a large number of OT neurons projecting to the posterior pituitary ${ }^{17}$ and can be accessed with minimum damage to the other hypothalamic regions. Hereafter, for simplicity, we refer to OT neurons located in the PVH as OT neurons. Optical fiber was implanted above the PVH of sexually naïve OT-Cre; Ail62 double heterozygote female mice. These mice were then crossed with stud male mice, and $\mathrm{Ca}^{2+}$ imaging was performed. Post-hoc histochemical analyses confirmed the fiber location (Fig. 1a). The GCaMP6s-expressing neurons were grossly healthy with no apparent morphological abnormalities (Fig. 1b). GCaMP6s was selectively expressed by the OT neurons with a specificity of $89.8 \pm 0.8 \%$ (mean \pm standard error of mean). The efficiency of GCaMP6s expression was rather modest: $59.0 \pm 1.0 \%$ OT neurons were GCaMP6spositive (Fig. 1b, c). This suboptimal efficiency might reflect the stochastic inactivation of the tetracycline response element (TRE) promoter in the Ai162 line.

To monitor OT neural activities during parturition, we applied an apparatus to capture the $\mathrm{Ca}^{2+}$ dynamics together with video records of the side and bottom views of the mouse cage during parturition (Supplementary Movie 1). We habituated the female mice to the apparatus and started recording from the evening before the expected parturition day. Parturition in our experimental condition occurred mostly from Zeitgeber 
Fiber photometry of maternal oxytocin pulses by Yukinaga et al.

time (ZT) 19 to ZT 1 (Fig. 1d), where ZT 0 is the onset of the light phase. It lasted for one to a few hours, with a median interval of about $20 \mathrm{~min}$ for each pup delivery (Fig. 1e). Fiber photometry data showed rhythmical pulsatile episodes of $\mathrm{Ca}^{2+}$ activities of OT neurons (hereafter referred to as OT-PAs for simplicity) that started about 10 min before each delivery, whereas no such episodes were observed in the 405-nm channel representing a non-calcium-dependent signal (Fig. 1f, g, Supplementary Fig. 1). From the side view, we observed abdominal contractions of the mother (Fig. 1f) occurring 10$15 \mathrm{~s}$ after the OT-PAs. The number of OT-PAs before each delivery was often 6-8, but this varied substantially, even among individual deliveries of pups by a single mother (Supplementary Fig. 1). In a heat map representation, high $\mathrm{Ca}^{2+}$ signals were clustered before the delivery of pups (Fig. 1h), while some intensive signals after the delivery of pups likely reflected the delivery of placental materials. These data illuminate the temporal dynamics of OT-PAs in the course of spontaneous parturition in mice.

\section{Activities of OT neurons during lactation}

We also recorded the $\mathrm{Ca}^{2+}$ dynamics of OT neurons of female mice in various reproductive conditions: nonpregnancy, during pregnancy and lactation, and after weaning of offspring (Supplementary Fig. 1a). No apparent OT-PAs were observed in the resting state of nonpregnant and late pregnant female mice. In sharp contrast, during lactation, when the postpartum mother mice were taking care of pups, we detected remarkable OT-PAs that disappeared immediately after weaning. These observations motivated us to characterize OT-PAs further in lactating mothers.

We were able to monitor OT neural activities overnight without affecting breastfeeding. A representative trace in Fig. 2a shows photometry data from postpartum day 12 (PPD12) mothers for 12 continuous hours. OT neurons often exhibited a cluster of OT-PAs throughout the recording session. In this trace, we detected 62 OT-PAs, with highly stereotyped height and full-width at half-maximum (FWHM) of individual pulses (Fig. 2b-d). By contrast, the inter-peak interval substantially varied; the majority clustered around $270 \mathrm{~s}$, yet small fractions ranged widely, from $20 \mathrm{~min}$ to close to $1 \mathrm{~h}$ (Fig. 2e).

To determine the minimum number of pups required for OT-PAs, we sequentially changed the number of pups (Supplementary Fig. 2b). The OT-PAs completely disappeared upon the removal of pups, and only one pup was insufficient. By contrast, we found that two to three pups or more can evoke stereotyped OT-PAs without a clear correlation between the number of pups and the height of the peaks. These observations support the notion that the observed OT-PAs are related to the milk ejection reflex. 
bioRxiv preprint doi: https://doi.org/10.1101/2021.07.26.453888; this version posted July 26, 2021. The copyright holder for this preprint (which was not certified by peer review) is the author/funder, who has granted bioRxiv a license to display the preprint in perpetuity. It is made available under aCC-BY-NC-ND 4.0 International license.

Fiber photometry of maternal oxytocin pulses by Yukinaga et al.

When we chronically monitored OT-PAs in the early and later lactation periods, we noticed that individual peaks became much higher as mothers experienced lactation. Therefore, we systematically compared the OT-PAs in PPD1 and PPD12-16. Because the intensities of OT-PAs observed in each mother varied considerably, probably because of the variable optical fiber location relative to the $\mathrm{PVH}$, the exact value of $\Delta \mathrm{F} / \mathrm{F}$ could not be compared among different animals. We therefore analyzed the fold change of the same animal from PPD1 to PPD12-16 and found that the height of the peaks was almost doubled in the PPD12-16 compared with the PPD1 mothers (Fig. 2f, g). The pulse duration (defined by FWHM) was also slightly but significantly increased in PPD12-16 compared with the PPD1 mothers. Enhanced OT-PAs in the experienced mothers may have been caused by stronger nipple sucking by elder pups. Alternatively, this change may be intrinsic to maternal OT systems independent of the sucking skills of pups. To distinguish these two possibilities, we cross-fostered pups between PPD1 and PPD12-16 mothers. The height of OT-PAs would change after swapping if the pups were the determinant. We found that the OT-PAs in PPD1 and PPD12-16 mothers remained unchanged regardless of the age of pups (Fig. $2 \mathrm{~h}-\mathrm{j}$ ). Therefore, enhanced OT-PAs in PPD12-16 mothers are not simply due to the greater nutritional requests by pups, which suggests the presence of plasticity in the OT neural system. This can lead to a more efficient milk ejection reflex as pups grow.

If the OT-PAs were enhanced by lactation experience, how would they behave in secundiparous mothers who nurse their second litters after weaning the first ones? In a small number of cases, we chronically monitored the same female mice throughout the first (\#1) and second (\#2) postpartum periods. Enhanced OT-PAs established by PPD12 ${ }^{\# 1}$ returned to baseline at PPD1 ${ }^{\# 2}$ and increased again by PPD12 $2^{\# 2}$ (Supplementary Fig. 3). This observation not only supports the notion that the lactation-dependent enhancement of OT-PAs is reset by each parturition, but also excludes the possibility that enhanced OTPAs are simply due to greater expression levels of GCaMP6s with aging. Collectively, our data reveal the temporal dynamics of OT-PAs during the milk ejection reflex.

Lastly, we compared the waveforms of OT-PAs during parturition (Fig. 1g) and lactation (Fig. 2b). In the same female mice (Supplementary Fig. 3c, d), the average FWHM was $6.8 \pm 1.4 \mathrm{~s}$ during parturition and $2.7 \pm 0.2 \mathrm{~s}$ at PPD1 of lactation. By contrast, the average peak height during parturition was only $29 \pm 7 \%$ that in PPD1; therefore, the OT-PAs in lactation were steeper. The inter-peak interval was much shorter during parturition; the median OT-PA interval was $50.3 \mathrm{~s}$ before delivery (Supplementary Fig. 1d) and about $270 \mathrm{~s}$ during lactation (Fig. 2e). These data reveal that the shape and interval of OT-PAs significantly differed between parturition and lactation, suggesting that the 
Fiber photometry of maternal oxytocin pulses by Yukinaga et al.

neural circuit mechanisms that generate OT-PAs change quickly from the peri-partition to the lactation phase.

\section{Input neural circuit mapping to OT neurons}

How are OT-PAs generated or modulated by afferent neural circuitry? Mapping monosynaptic input to OT neurons would be the first step to address this question. Therefore, we applied Cre-dependent rabies-virus-mediated trans-synaptic tracing ${ }^{27,30}$ to OT neurons. To map long-distance input to OT neurons efficiently, we injected Cre-dependent adenoassociated virus (AAV) vectors for CAG-FLEx-TC ${ }^{b}\left(T V A-m C h e r r y^{b r i g h t}\right)$ and CAG-FLEx$R G$ (rabies glycoprotein) into the PVH of sexually naïve OT-Cre female mice. We then crossed them with stud male mice and injected rabies $d G-G F P+E n v A$ into the PVH of age-matched nonpregnant female mice and PPD1 mothers (Fig. 3a) for a side-by-side comparison.

As the $\mathrm{TC}^{\mathrm{b}}$-mediated trans-synaptic tracing method is known to cause Creindependent nonspecific infection of rabies $d G-G F P+$ EnvA in the local injection site ${ }^{27}$, which compromises the local circuit analysis, we first evaluated the degree of this local background labeling. We injected AAVs and rabies virus by following the same scheme mentioned above into the non-Cre expressing wild-type mice (Supplementary Fig. 4a-c). We detected $385.8 \pm 76.4$ GFP expressing (GFP+) cells as nonspecific rabies labeling, the vast majority of which were located in the PVH (72\%) and paraventricular thalamus (PVT, $18 \%$ ). No long-distance labeling was found beyond $500 \mu \mathrm{m}$ in the injection site. Other hypothalamic nuclei surrounding the $\mathrm{PVH}$, such as the anteroventral periventricular nucleus (AVPV) and dorsomedial hypothalamus (DMH), contained no or only a little nonspecific labeling. Based on these data, we excluded PVH and PVT from the analysis of long-range input to OT neurons.

Overall, the number of starter cells (i.e., those labeled with TVA-mCherry and rabies-GFP) was comparable in nonpregnant and lactating female mice (Fig. 3b, c), consistent with a previous report showing no major changes in the number of OT neurons upon lactation in rat $^{31}$. The majority of pre-synaptic neurons were located in the broad hypothalamic areas (Fig. 3d, e) with prominent input structures, including the medial preoptic nucleus (MPN), supraoptic nucleus (SO), DMH, ventromedial hypothalamus (VMH), and arcuate hypothalamic nucleus (ARH). We also noticed substantial input cells in the extra-hypothalamic areas, such as the lateral septum (LS), the bed nucleus of the stria terminalis (BST), and the periaqueductal gray of the midbrain (PAG). Input patterns were grossly similar between nonpregnant female mice and lactating mothers (Fig. 3e-g, Supplementary Table 1), with no statistically significant differences in any brain region; 
Fiber photometry of maternal oxytocin pulses by Yukinaga et al.

however, a weak tendency was seen for anterior brain regions to send more input to OT neurons in lactating mothers.

Next, we analyzed the cell type of input neurons. Brain slices containing six selected areas were stained by in situ hybridization (ISH) with an excitatory or inhibitory neural marker, $v G l u T 2$ or $v G A T$, together with immunostaining of rabies-GFP (Fig. 4). Input neurons originating from the AVPV and DMH were mixed with $v G l u T 2+$ and $v G A T+$ subpopulations, whereas those from the LS, BST, and ARH were mostly GABAergic and those from the VMH were mostly glutamatergic. No difference in the fraction of excitatory or inhibitory neurons was observed between nonpregnant female mice and lactating mothers.

As $\mathrm{TC}^{\mathrm{b}}$-based trans-synaptic tracing contained substantial local background labeling, to characterize local connectivity within the PVH more accurately, we utilized a mutant TVA receptor ${ }^{27}$ to reduce the nonspecific labeling to zero (Fig. 5a). We injected AAV2 $C A G-F L E x-T C^{66 T}$ and AAV2 $C A G-F L E x-R G$, followed by rabies $d G-G F P+$ EnvA, into the PVH of OT-Cre mice. As a negative control, we omitted the AAV CAG-FLEx$R G$ cassette and found no neuron expressing GFP alone ( $n=5$ animals), confirming that there was no background labeling by this system. As shown in Fig. 3, we compared labeling patterns following rabies injection into age-matched nonpregnant female mice and PPD1 mothers. We detected $125.0 \pm 30.1$ and $115.7 \pm 36.4$ starter cells in nonpregnant and lactating female mice, respectively, from which rabies-GFP spread to local PVH neurons (Fig. 5b, c), showing abundant local PVH input to OT neurons. To characterize the cell type of GFP-labeled neurons, we detected mRNA for OT and its related peptide hormone vasotocin ${ }^{32}$ (VT; also known as arginine-vasopressin), together with immunostaining of rabies-GFP (Fig. 5d). Although these two hormones were encoded by highly homologous genes, we carefully designed ISH probes to distinguish $O T$ and $V T$ (Supplementary Fig. 4d, e). In nonpregnant female mice, $6.2 \pm 1.0 \%$ of GFP + neurons were $O T+$, and $17.9 \pm 2.4 \%$ were $V T+$, showing intensive connectivity among neurons producing posterior pituitary hormones. In lactating females, OT-to-OT connection tended to increase $(13.7 \pm 2.4 \%, \mathrm{p}=0.023$ by $t$-test $)$, whereas no change was seen in VTto-OT connection ( $\mathrm{p}=0.40$ by $t$-test, Fig. $5 \mathrm{e}$ ). As VT and OT neurons account for only $20 \%-30 \%$ of GFP+ neurons in the PVH, other types of neurons are likely to contribute to the regulation of OT neurons via the local circuits. Collectively, our study illuminates the presence of intensive local synaptic connectivity within the PVH.

In summary, our input circuit mapping forms a basis to characterize afferent neural circuitry to OT neurons that underlie various OT-mediated biological processes, including parturition, lactation, and social bonding ${ }^{3}$. 
Fiber photometry of maternal oxytocin pulses by Yukinaga et al.

\section{Pharmaco-genetic manipulations of maternal OT pulses}

The mono-synaptic input map to the OT neurons (Figs. 3-5) allows us to ask how maternal OT-PAs (Figs. 1, 2) may be shaped by afferent circuitry. As a proof-of-principal, we combined cell-type-specific pharmaco-genetic manipulations of a defined presynaptic structure with OT-Cre-mediated fiber photometry recordings of OT-PAs. We selected the inhibitory neurons located in the septum and BST (Fig. 4b) because they are the prominent long-distance input to OT neurons. To target inhibitory neurons selectively, we utilized a small enhancer sequence of human $D l \times 5 / 6$ intergenic region ${ }^{33}$ to drive $\mathrm{hM} 3 \mathrm{Dq}$ or hM4Di for activation or inactivation of the targeted neurons ${ }^{34}$, respectively. After validating the AAV vectors to be specific to inhibitory neurons in the motor cortex (Supplementary Fig. 5), we injected AAV1 $D l x-h M 3 D q$ or AAV5 Dlx-hM4Di into the septum and BST regions of OT-Cre; Ai162 female mice, followed by the implantation of an optical fiber above the PVH (Fig. 6a). After parturition, we performed a 6-h photometry recording twice in PPD6-13 mothers. On the first day, either saline control or clozapine-N-oxide (CNO) was intraperitoneally injected $2 \mathrm{~h}$ after the onset of the imaging session. On the second day, we replaced saline and CNO for counterbalancing. Post-hoc histochemical analyses confirmed intensive hMD3q or hM4Di expression, inferred by co-expressing nuclear-dTomato marker, in the BST and septum (Fig. 6b). In the hM3Dq-expressing female mice, we observed a drastic reduction of OT-PAs following CNO injection to activate inhibitory neurons in the septum and BST. This effect lasted for several hours and returned to baseline by the next day. By contrast, no such change was found in control saline-injected trials (Fig. 6c, e, f). In the hM4Di-expressing female mice, CNO injection did not drastically change OT-PAs (Fig. 6d, e, f). In both $\mathrm{hM} 3 \mathrm{Dq}$ and hM4Di experiments, the waveform of individual remaining OT-PAs was not changed by $\mathrm{CNO}$ application. These data suggest that the inhibitory neurons in the septum and BST are important modulators of OT-PAs.

\section{Discussion}

Parturition and lactation are complex biological processes involving bidirectional interactions between the maternal uterus/mammary gland and brain, abnormalities in which lead to mortality among mothers and children, particularly in low-resource settings. Despite the clinical importance and direct involvement in the quality of life of postpartum women, basic neuroscience studies of parturition and lactation processes remain limited. Therefore, we discuss the technical advances and new findings provided by the present study. 
Fiber photometry of maternal oxytocin pulses by Yukinaga et al.

\section{Cell-type-specific recording of maternal OT neural activities}

Cell-type-specific recording and manipulation of OT systems ${ }^{3}$ began to be utilized in studies of OT-mediated emotions and social behaviors. For example, a mini-enhancermediated AAV vector for cell-type-specific optogenetic targeting of OT neurons was first introduced in the context of the anxiolytic effects of OT in $\mathrm{rat}^{31}$. Cre-dependent optogenetic activation of OT neurons was also used to facilitate maternal behaviors ${ }^{35}$. To establish effective sites of OT, cell-type-specific loss-of-function of OTR or brain regionspecific ablation of OTR-expressing neurons was used in studies of social reward learning $^{36}$, sex recognition ${ }^{37}$, sex preference ${ }^{38}$, and social fear ${ }^{39}$. The present study expands the scope of cell-type-specific recording of OT neural activities ${ }^{19-22}$ to the classical maternal OT functions during parturition and lactation.

By using fiber photometry, we revealed rhythmical OT-PAs that only appeared during parturition and lactation. Our data generally support classical single-unit recording of putative OT neurons in rats ${ }^{1}$. For example, OT-PAs were initiated about $10 \mathrm{~min}$ before the delivery of the first pup (Fig. 1), in line with data from parturient rats ${ }^{40}$. The interpeak interval of OT-PAs during the milk ejection reflex was about $270 \mathrm{~s}$ (Fig. 2e), close to the $300 \mathrm{~s}$ described in rats ${ }^{9,12}$. Three or more pups were needed to maintain the PAs during lactation (Supplementary Fig. 2b), similar to the finding that seven or more pups were required in rats ${ }^{10}$. This difference may be due to the experimental conditions (awake versus anesthetized) and/or species. Our chronic imaging also provides new findings. For example, OT-PAs often formed clusters that lasted for $30 \mathrm{~min}$ to a few hours, followed by a period of non-OT-PAs for 20 min to $1 \mathrm{~h}$ (Fig. 2a). As such temporal dynamics likely reflect complex mother-infant interactions during lactation, this system is expected to facilitate the identification of internal and environmental factors that modify the quality of lactation in the future.

We also found the enhancement of individual OT-PAs, without affecting the inter-peak interval or waveform, from PPD1 to PPD12-16 mothers (Fig. 2). As the age of pups did not significantly affect the intensity of the OT-PAs, we speculate that this plasticity is mostly autonomous to the OT system of mothers. What would be the underlying mechanisms? In one scenario, the number of OT neurons forming OT-PAs is not changed from PPD1 to PPD12-16, but each neuron fires more. Alternatively, new OT neurons are recruited to the OT-PAs as mothers experience lactation. To distinguish these two possibilities, we must await future studies utilizing a miniaturized microscope ${ }^{41}$ for single-cell resolution imaging of OT-PAs. As we found that the height, inter-peak interval, and waveform of PAs substantially differed between parturient and lactating females, it 
Fiber photometry of maternal oxytocin pulses by Yukinaga et al.

would also be interesting to ask if OT neurons forming the OT-PAs during parturition also work for the milk ejection reflex, or if distinct sub-populations of OT neurons exist for delivery and lactation.

\section{Mono-synaptic input map to OT neurons and regulation of the OT-PAs}

Another important aspect of the present study is the identification of direct pre-synaptic partners of OT neurons by using a rabies-virus mediated trans-synaptic tracing technique ${ }^{27,30}$. While devising this study, we noticed that a few other papers and preprints had started to report similar trans-synaptic tracing data in rats and mice in different biological contexts ${ }^{20,22,42}$. Overall, our data are consistent with those studies, as the majority of input to OT neurons was located in broad hypothalamic nuclei. Our data uniquely expand the scope of circuit analysis to lactating mothers and provide detailed and quantitative information on pre-synaptic structures and cell types (Figs. 3 and 4). In addition, we show the presence of local OT-to-OT and VT-to-OT connections (Fig. 5). One interesting difference between our data and the input map to OT neurons in rats $^{20}$ is the abundance of cortical top-down input from the prelimbic and cingulate cortex, which was observed in rats ${ }^{20}$, but not in our data or another data set in mice ${ }^{42}$. An intriguing possibility is that this is a species-specific difference and that rat OT neurons can receive more intensive cortical top-down regulation. There is a technical concern, however, in the specificity of the tracing system utilized in Ref. 20, in which rabies-glycoprotein (which defines the neurons able to spread rabies virus) was not genetically restricted to OT neurons. More careful studies to compare input patterns between mice and rats are therefore essential in the future.

As the morphological changes in glia-OT neuron associations during lactation have been well documented ${ }^{43}$, we initially expected a drastic reorganization in input to OT neurons in mothers compared with nonpregnant female mice. However, our data suggest widespread and nuanced adjustments of connections (Figs. 3 and 5) and do not support a model assuming a drastic rearrangement of a small number of neural circuit nodes that underpins the temporal specificity of maternal OT-PAs.

Nevertheless, our high-resolution input map to OT neurons is a useful resource to characterize afferent circuitry functions to OT neurons. To show this utility by pharmaco-genetic manipulations, we identify an important modulatory function of inhibitory neurons in the septum and BST (Fig. 6). Our data not only confirm classical lesion and electronic stimulation data in rats $^{25,44}$, but also expand the scope to cell-typespecific and reversible manipulations of OT-PAs. As diverse signals such as vomeronasal input $^{45}$, stress, and anxiety ${ }^{46}$ are mediated by the septum and BST, it is interesting to ask 
Fiber photometry of maternal oxytocin pulses by Yukinaga et al.

if septum/BST input to OT neurons is indeed involved in the acute physical and/or mental stress-induced impairment of the milk ejection reflex, a phenomenon often reported in postpartum women ${ }^{47}$. More generally, our data form the basis for comprehensive circuit characterizations of various input nodes. We expect that viral genetic manipulations of upstream neural activities or gene functions will illuminate the circuit mechanisms by which maternal OT-PAs are shaped. 
Fiber photometry of maternal oxytocin pulses by Yukinaga et al.

\section{Main Figures}

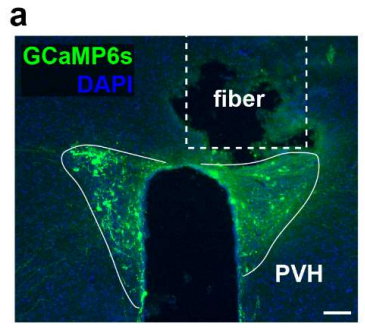

C

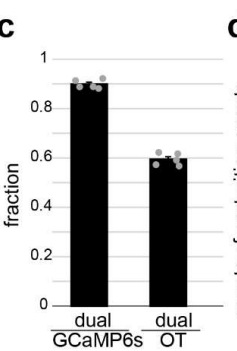

d

f

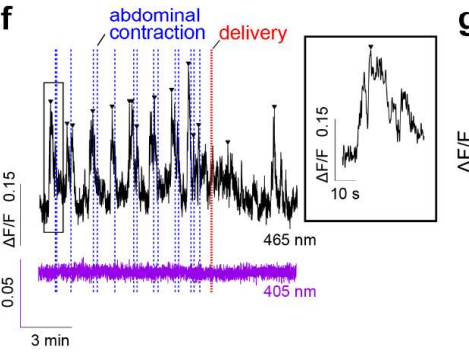

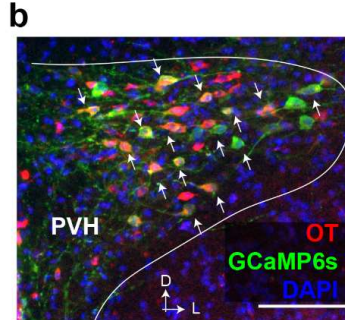

e

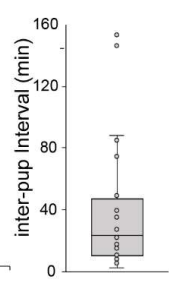

g

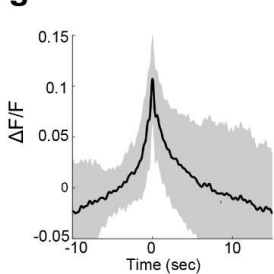

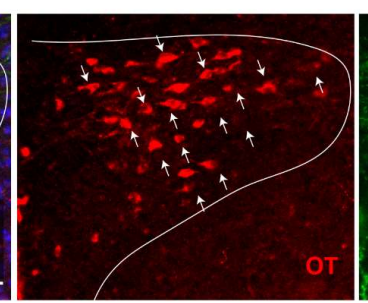

h

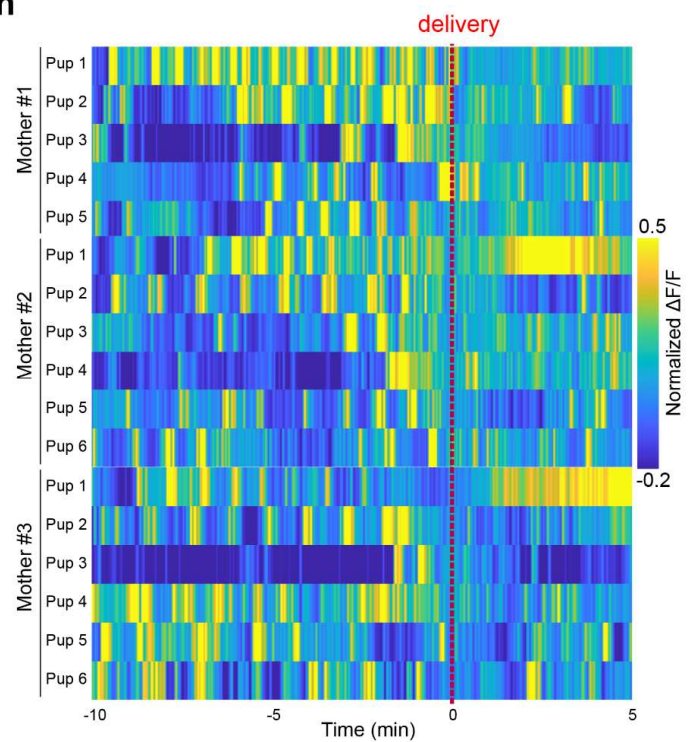

Fig. 1 Fiber photometry recording of OT neurons during parturition

a Representative coronal brain section showing the location of the optical fiber and expression of GCaMP6s in the PVH. b Typical example of a 30- $\mu$ m coronal section showing PVH stained by anti-OT (red) and anti-GFP (for GCaMP6s, green) antibodies counterstained with DAPI (blue). Arrows indicate some of the GCaMP6s-positive cells. Of note, most GCaMP6s-positive cells expressed OT, whereas a substantial number of OT neurons were GCaMP6s-negative. D, dorsal, L, lateral. c Quantification of specificity (dual/GCaMP6s) and efficiency (dual/OT). $n=5$ mice each. d Histogram showing the temporal distribution of the initiation of parturition in our experimental setting. $\mathbf{e}$ Quantification of the pup delivery interval. The center line shows median; box limits represent upper and lower quartiles. $\mathbf{f}$ Representative peri-event photometry traces of the 405-nm channel (non-calcium-dependent background, purple) and the 465-nm channel (calcium-dependent GCaMP6s signals, black) showing -10 min to +5 min relative to delivery. OT-PAs are indicated by arrowheads, and the timing of abdominal contractions and pup delivery are represented by blue and red vertical dotted lines, respectively. Of note, this sample (mother \#2-pup 2) corresponds to the data shown in Supplementary Movie 1. The inset shows the high magnification view of the boxed area. $\mathbf{g}$ An averaged 
Fiber photometry of maternal oxytocin pulses by Yukinaga et al.

peri-event trace of 37 individual OT-PAs observed in mother \#2 from -10 min to delivery (Supplementary Fig. 1). The shadow represents the standard deviation (SD). $\mathbf{h}$ Colored heat map representation of normalized $\Delta \mathrm{F} / \mathrm{F}$ from $-10 \mathrm{~min}$ to +5 min relative to delivery for three mothers. For each mother, the data of the first 5-6 deliveries are analyzed, because after the sixth delivery, the nest became too crowded to analyze the timing of the birth. Scale bar, $100 \mu \mathrm{m}$. 
Fiber photometry of maternal oxytocin pulses by Yukinaga et al.

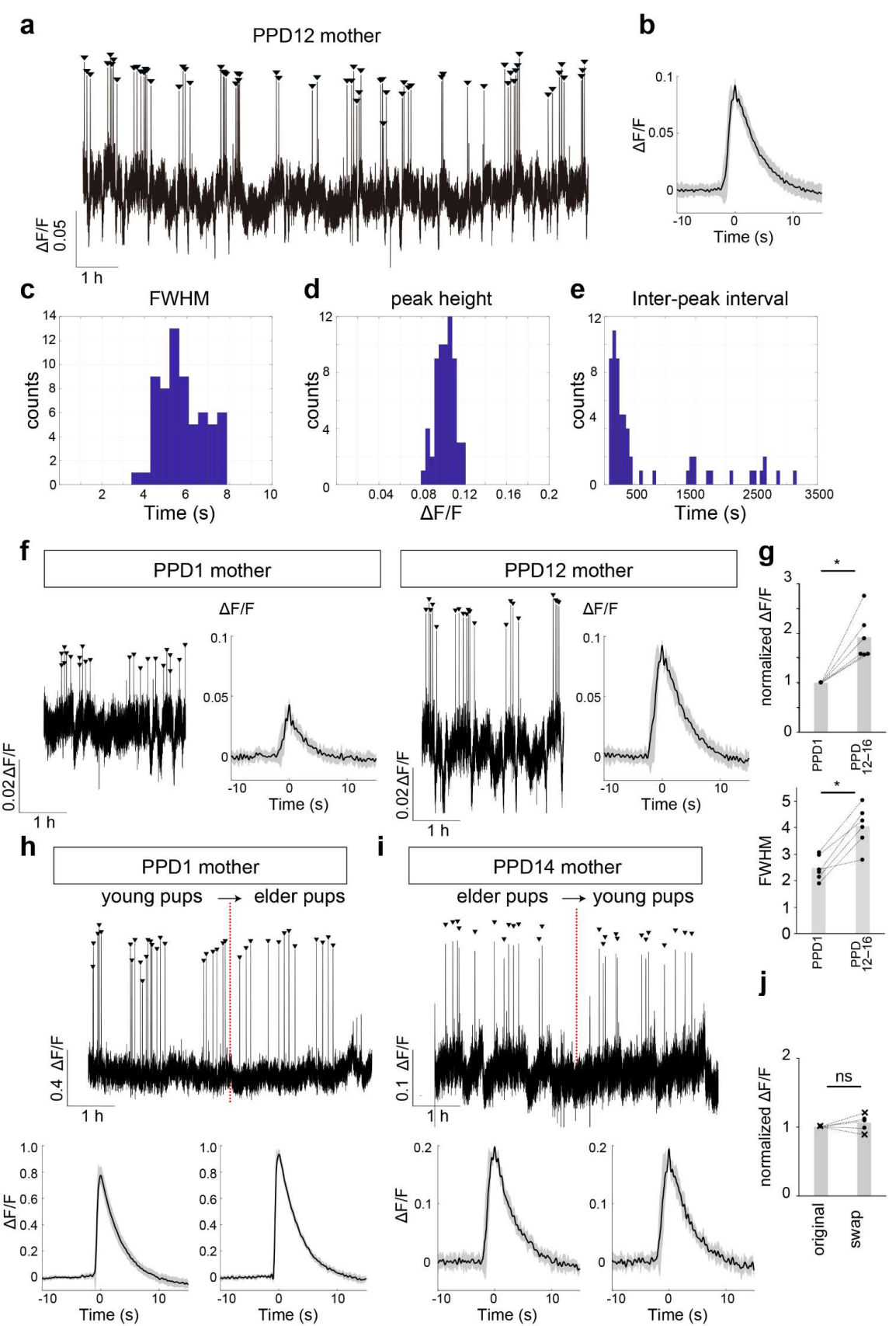

Fig. 2 Fiber photometry recording of OT neurons during lactation

a Representative 12-h continuous fiber photometry trace from a PPD12 mother containing 62 OT-PAs. b Averaged peri-event trace of these OT-PAs, with shadows showing the SD. c-e Histograms showing full-width at half-maximum (FWHM, c), peak height (d), and inter-peak interval (e) from the raw data shown in (a). f Representative examples of 3-h traces of fiber photometry data obtained from PPD1 and PPD12 mothers. Peri-event traces of individual OT-PAs are shown on the right of the raw traces, with shadows showing the SD. g (top) Peak height of the OT-PAs was calculated for each 
Fiber photometry of maternal oxytocin pulses by Yukinaga et al.

animal and shown as fold change from PPD1 to PPD12-16 of the same animal. OT-PAs were significantly enhanced ( $*, p=0.028$ by two-sided Wilcoxon signed-rank test). (bottom) FWHM of the OT-PAs from PPD1 to PPD12-16 mothers (*, $\mathrm{p}=0.028$ by Wilcoxon signed-rank test). $n=6$ mothers. $\mathbf{h}$, $\mathbf{i}$ Representative examples of 3 -h traces of fiber photometry data obtained from PPD1 (h) or PPD14 (i) mothers during lactation. In the middle of the recording session, the original pups were removed and foster pups were introduced. Averaged peri-event traces of OT-PAs are shown at the bottom, with shadows showing the SD. $\mathbf{j}$ Quantification of the fold-change of peak height before (original) and after swapping pups. No significant difference was found ( $p=0.50$ by two-sided Wilcoxon signed-rank test). PPD1 mothers (shown as $\times$ symbols), $n=2$, and PPD12-16 mothers (shown as closed circles), $n=3$. Arrowheads represent OT-PAs. 
Fiber photometry of maternal oxytocin pulses by Yukinaga et al.
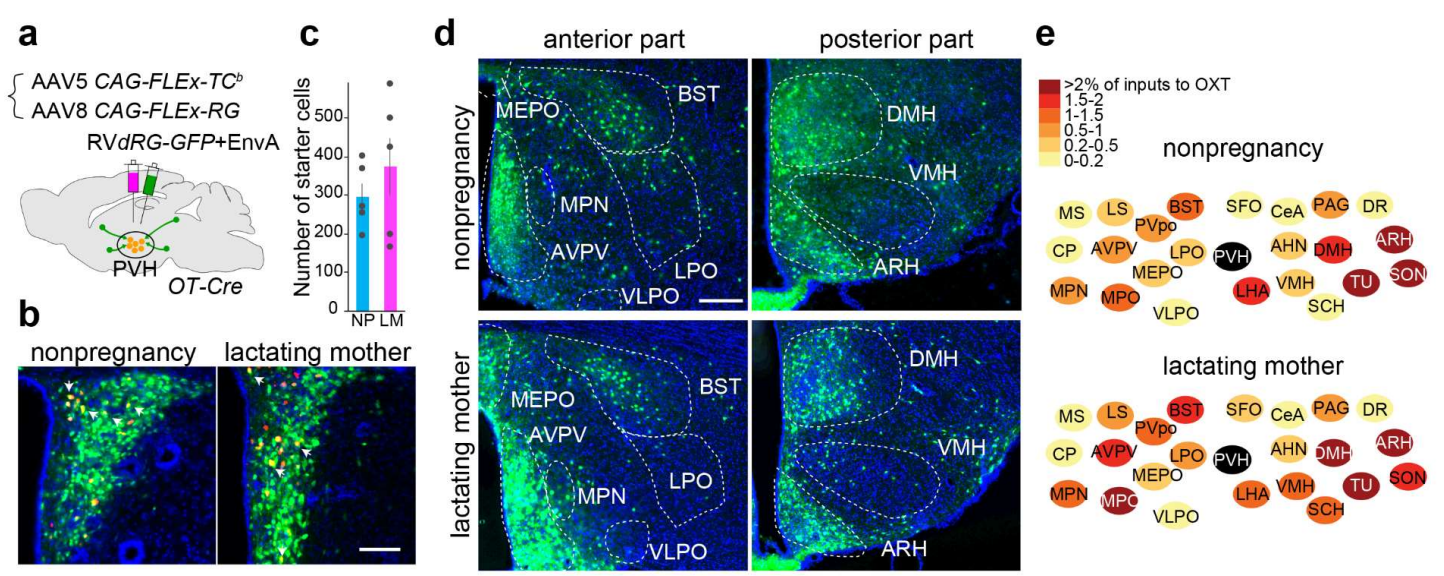

f
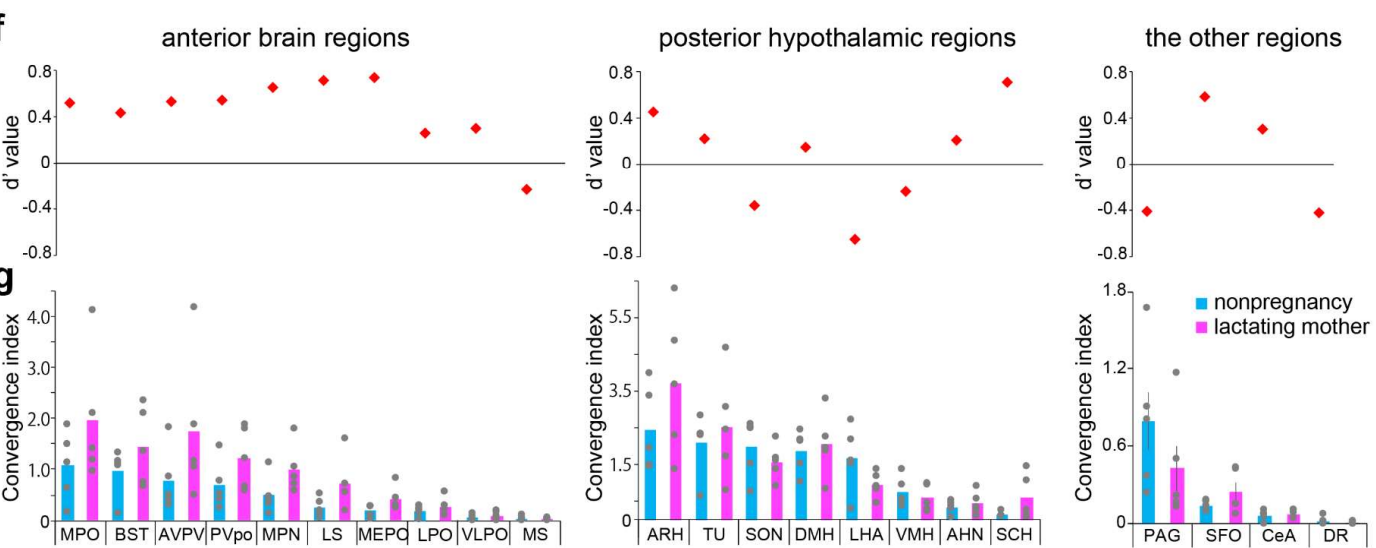

Fig. 3 Quantification of input cells to OT neurons in the postpartum female mice

a Schematics of the experimental design. b Coronal sections of OT-Cre tracing brains showing the starter cells (defined by co-expression of TVA-mCherry and rabies-GFP, some indicated by arrows) in the PVH. Scale bar, $100 \mu \mathrm{m}$. c Quantification of starter cells per animal. No significant difference was found between nonpregnancy (NP) and lactating mothers $(\mathrm{LM})$ by the Wilcoxon signed-rank test $(\mathrm{p}=0.48) . n=5$ each. d Representative coronal sections showing the distribution of pre-synaptic partners. Scale bar, $200 \mu \mathrm{m}$. e Schematic colored heat map representation of the brain regions that provided large average fractional inputs to OT neurons in the nonpregnant (top) and lactating (bottom) female mice. $\mathbf{f}-\mathbf{g}$ The $\mathrm{d}$ '-values and average convergence index (defined by the number of rabies-GFP + cells normalized to the number of starter cells) with individual animal data in gray. $n=5$ each. For abbreviations of brain regions, see Supplementary Table 2 . 
bioRxiv preprint doi: https://doi.org/10.1101/2021 07.26.453888; this version posted July 26, 2021. The copyright holder for this preprint (which was not certified by peer review) is the author/funder, who has granted bioRxiv a license to display the preprint in perpetuity. It is made available under aCC-BY-NC-ND 4.0 International license.

Fiber photometry of maternal oxytocin pulses by Yukinaga et al.

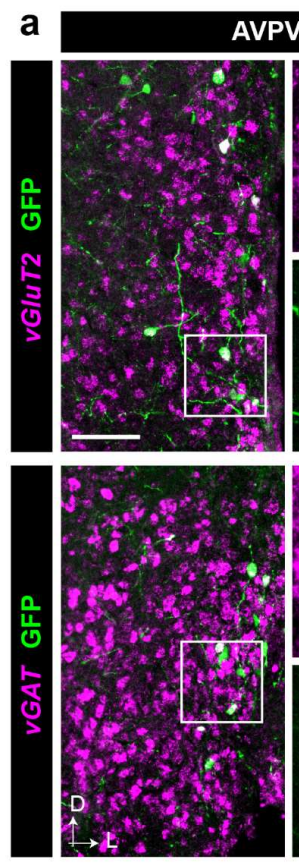

AVPV
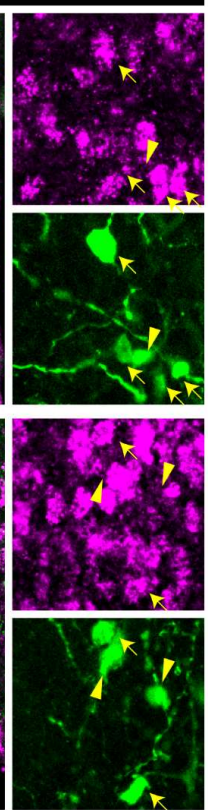

vBST
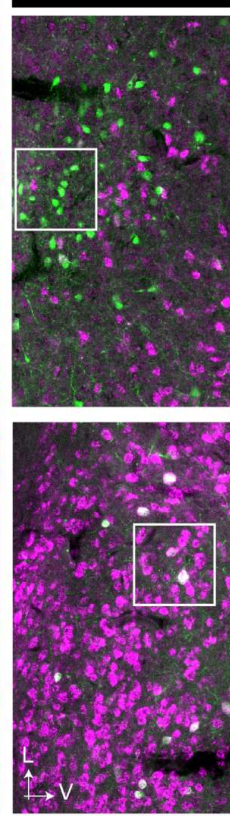

b
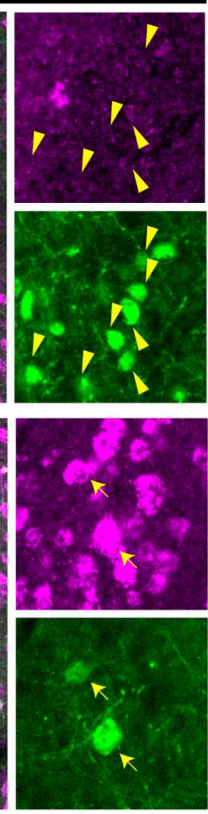

b (vGluT2+ GFP+) / GFP+

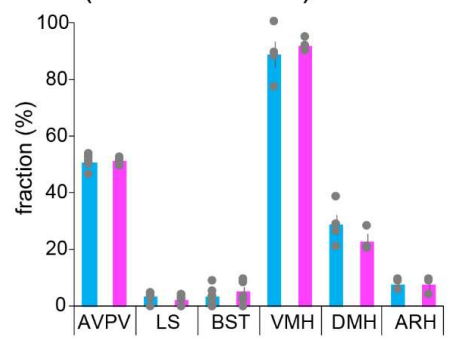

$(v G a t+G F P+) /$ GFP+

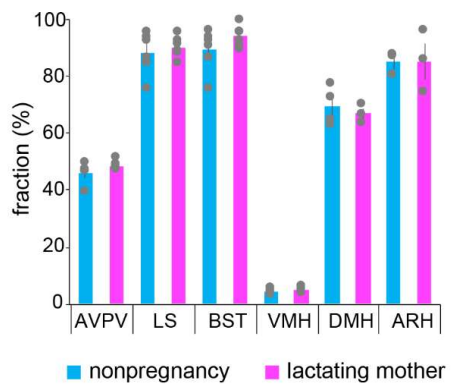

Fig. 4 Cell-type analysis of rabies-labeled neurons

a AVPV and ventral BST (vBST) coronal sections with $v G l u T 2$ or $v G A T$ in situ hybridization (red), along with anti-GFP immunostaining (green). Scale bar, $100 \mu \mathrm{m}$. High magnification images to the right of each image correspond to boxed areas in the low magnification images. Yellow arrows indicate markers and GFP double-labeled cells, whereas yellow arrowheads show examples of GFP-positive but marker-negative cells. D, dorsal, L, lateral, V, ventral. b Average fractions of GFP-positive cells that are vGluT2positive (top) or $v G A T$-positive (bottom) in the indicated brain regions. Error bars represent the SEM. Individual data are shown in gray. $n=3-6$ animals for each brain region. 
Fiber photometry of maternal oxytocin pulses by Yukinaga et al.
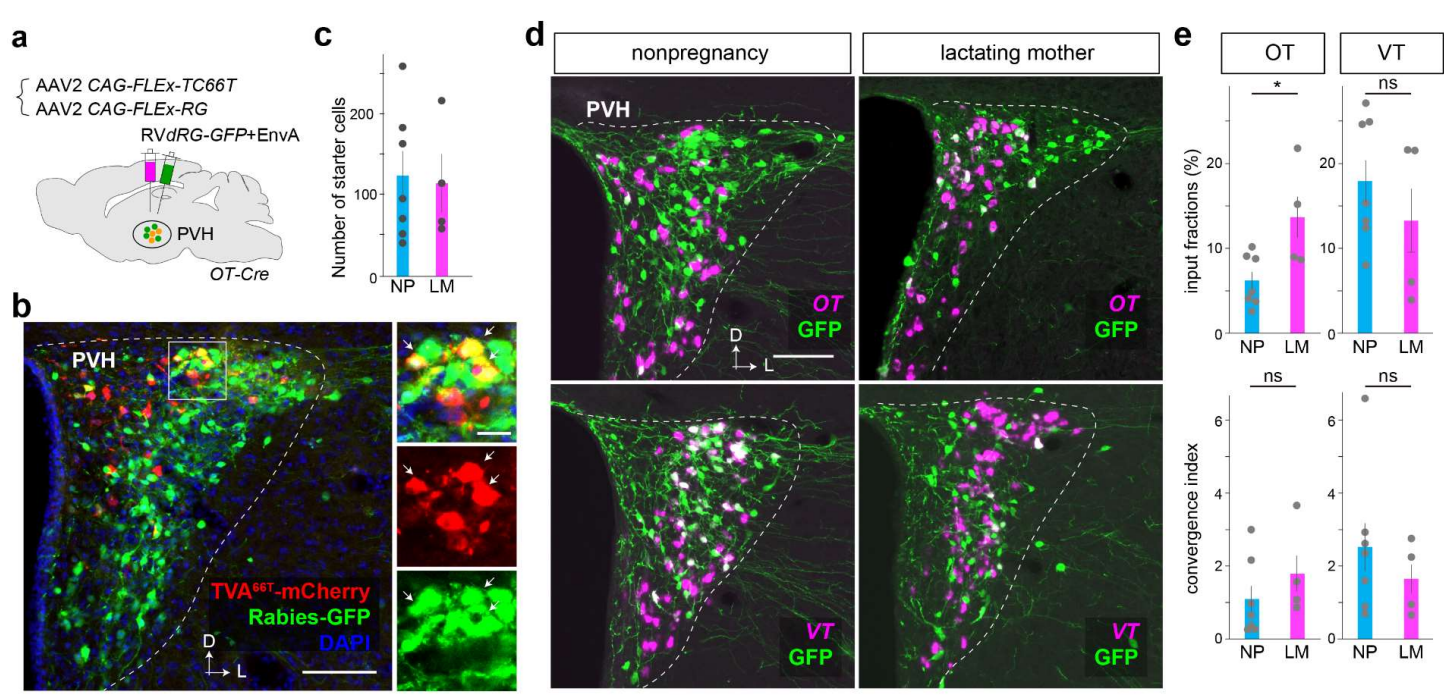

Fig. 5 Local circuit input to OT neurons within the PVH

a Schematics of the experimental design. b Typical coronal section showing the starter cells that co-expressed TVA-mCherry (red) and rabies-GFP (green) in the PVH. D, dorsal, L, lateral. High magnification images to the right exhibiting red, green, and superimposed images correspond to the boxed area in the low magnification image. Arrows indicate some of the starter cells. Scale bar, $100 \mu \mathrm{m}$ for low and $20 \mu \mathrm{m}$ for high magnification images. c Quantification of starter cells per animal. No significant difference was found between nonpregnant (NP) and lactating mothers (LM) by the Wilcoxon signed-rank test ( $\mathrm{p}=0.6) . n=7$ for NP and $n=3$ for LM. d Representative coronal sections with OT or vasotocin (VT) in situ hybridization (magenta), along with anti-GFP immunostaining (green). Scale bar, $100 \mu \mathrm{m}$. D, dorsal, L, lateral. e Average input fraction (top) and convergence index (bottom) of OT and GFP dual-labeled cells (left) or VT and GFP duallabeled cells (right). Error bars, SEM. Individual data are shown in gray. *, $p=0.023$ by $t$-test, ns, $\mathrm{p}>0.05$ by two-sided $t$-test. Of note, the fraction and convergence index of OTto-OT connections are underestimated because some pre-synaptic OT neurons may be recognized as starter cells. The estimated number of starter cells corresponded to $8.3 \pm$ $2.4 \%$ and $13.4 \pm 6.3 \%$ of OT + neurons in NP and LM samples, respectively ( $\mathrm{p}=0.52$ by the two-sided Wilcoxon signed-rank test). 
Fiber photometry of maternal oxytocin pulses by Yukinaga et al.
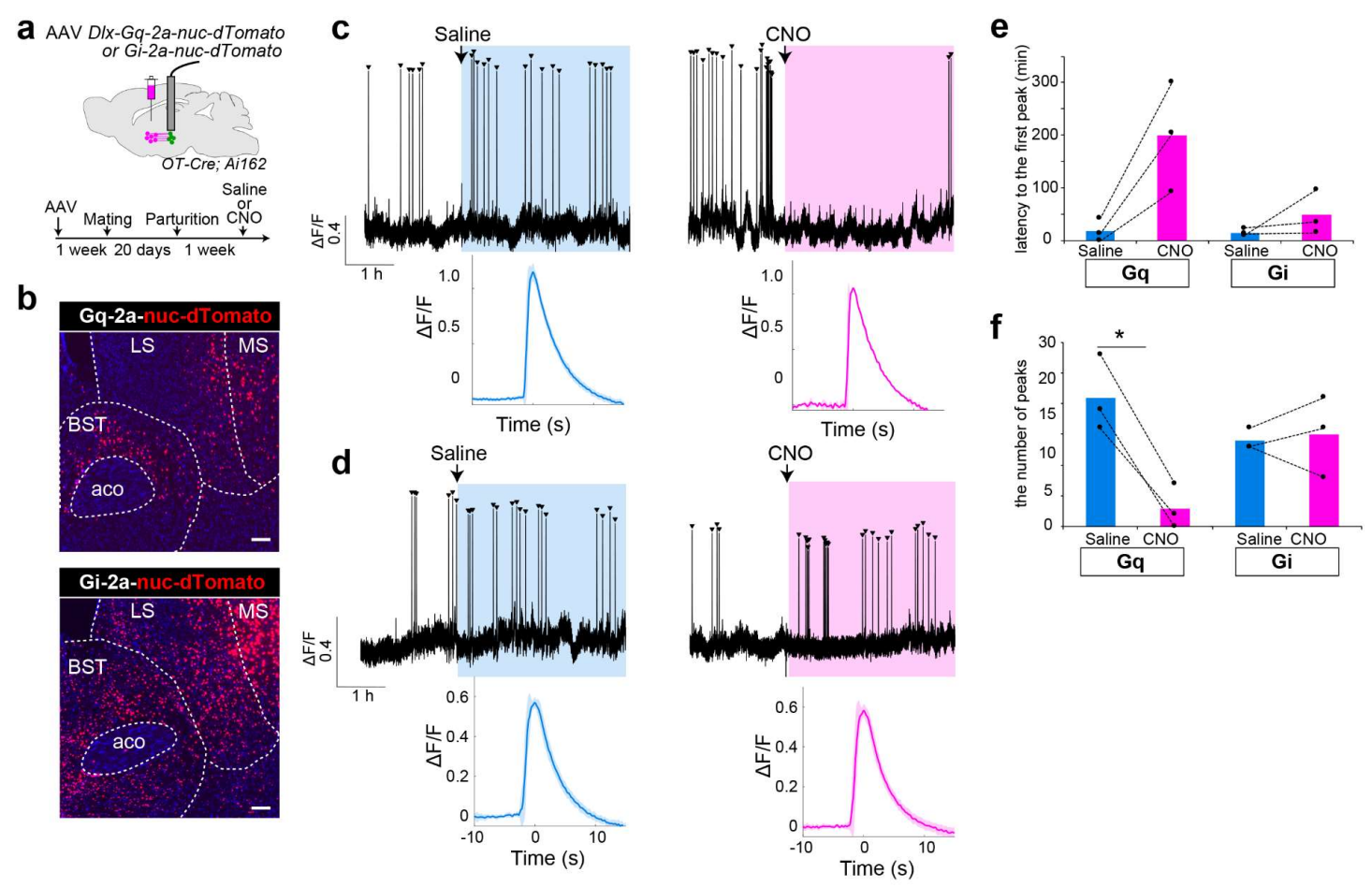

Fig. 6 Modulation of OT pulsatile activities by pharmaco-genetic manipulations

a Schematics of the experimental design and timeline of the experiments. b Representative coronal sections showing expression of hM3Dq (top) or hM4Di (bottom) inferred by the co-expressing nuclear-dTomato marker in the LS, MS, and BST. Scale bars, $100 \mu \mathrm{m}$. c, d Representative 5.5-h traces showing OT-PAs (arrowheads) in hM3Dqexpressing (c) or hM4Di-expressing (d) mothers. The timing of saline or CNO injection is indicated by the vertical arrows. Averaged peri-event traces of individual OT-PAs detected after injection of saline or CNO are shown at the bottom, with shadows showing the SD. e Quantification of latency to the first OT-PA following saline or CNO injection. In Gq-expressing animals, the latency tended to be elongated ( $p=0.057$ by two-sided paired $t$-test). f Quantification of the number of OT-PAs in $3.5 \mathrm{~h}$ following saline or CNO injection. $n=3$ for hM3Dq and $n=3$ for hM4Di. ${ }^{*}, \mathrm{p}<0.05$ by two-sided paired $t$-test. 
Fiber photometry of maternal oxytocin pulses by Yukinaga et al.

\section{Supplementary Figures}
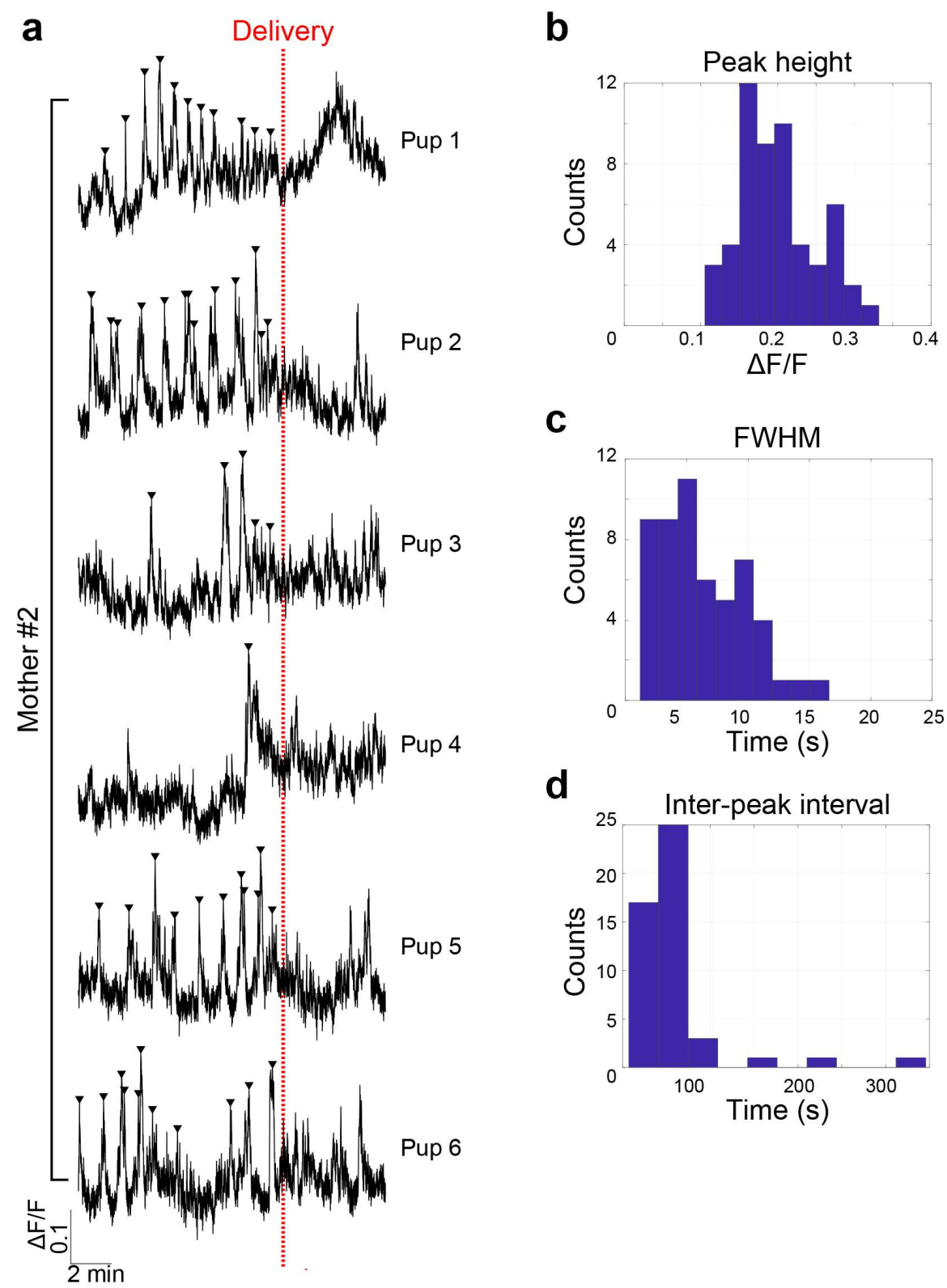

Supplementary Fig. 1 Raw data of $\mathrm{Ca}^{2+}$ traces during the parturition from a single female mouse, related to Fig. 1

a Peri-event photometry traces from $-10 \mathrm{~min}$ to $+5 \mathrm{~min}$ relative to the delivery (indicated by the red vertical line) of each pup from a single female mouse (corresponding to mother \#2 in Fig. 1). OT-PAs detected before each delivery are shown by arrowheads. b-d Histograms showing peak height (b), the full-width at half-maximum (FWHM, c), and the inter-peak interval (d). Peaks that occurred $10 \mathrm{~min}$ before each pup delivery from the raw data shown in (a) were used for the calculation. 
Fiber photometry of maternal oxytocin pulses by Yukinaga et al.

a

Non-pregnancy

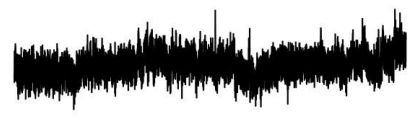

Pregnancy (gestation day 15)

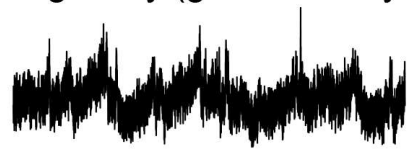

Lactation (postpartum day 9)

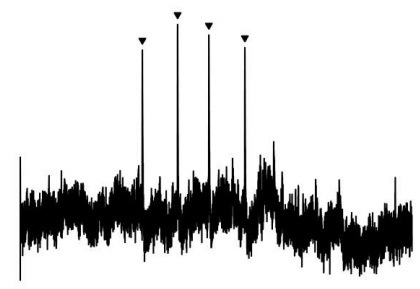

After weaning

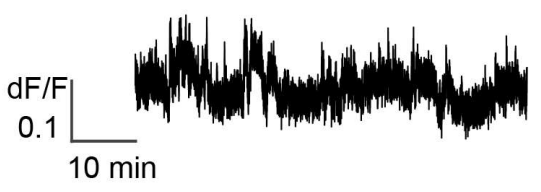

b

\# of pups $\quad 0 \quad 1$
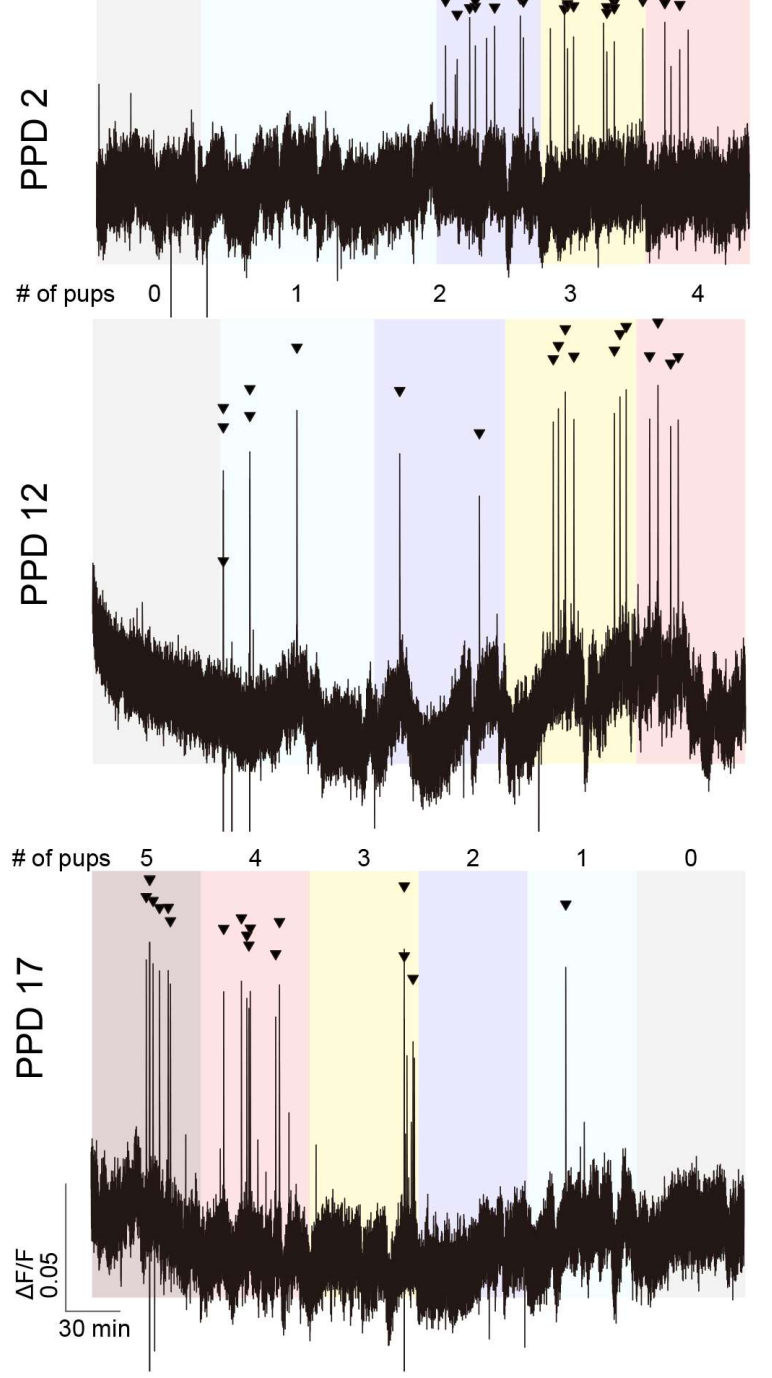

Supplementary Fig. 2 OT-PAs in various reproductive conditions and with different numbers of pups, related to Fig. 2

a Representative examples of individual 1-h traces of fiber photometry data obtained from different stages of female mice as indicated. b Three representative examples of OT neural activities during lactation at different postpartum days (PPD2, 12, or 17), when the number of pups was artificially changed from $0-4$ or 5 . Zero or one pup did not efficiently evoke OT-PAs; 2-3 pups were necessary (arrowheads). 
Fiber photometry of maternal oxytocin pulses by Yukinaga et al.
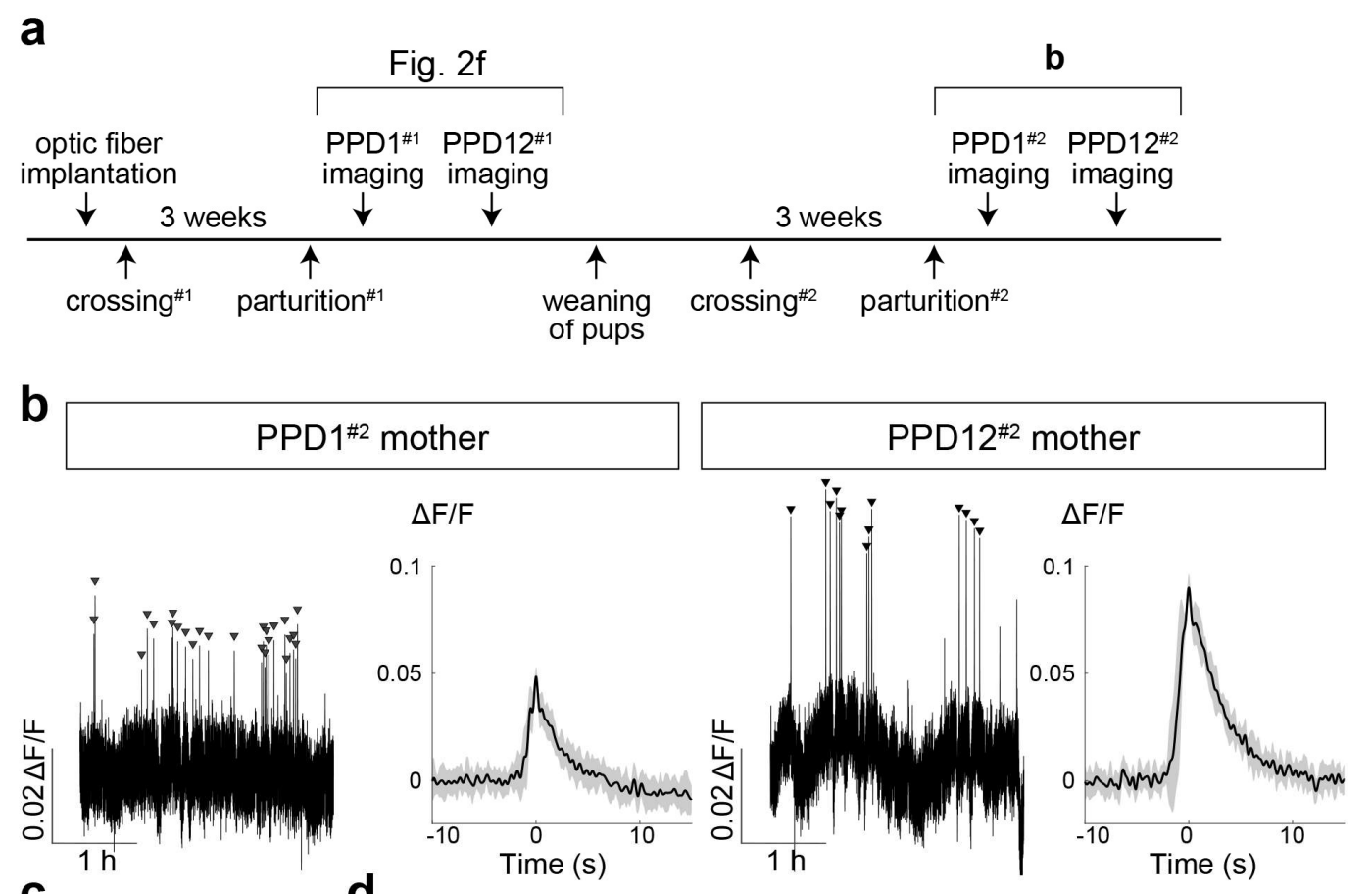

C
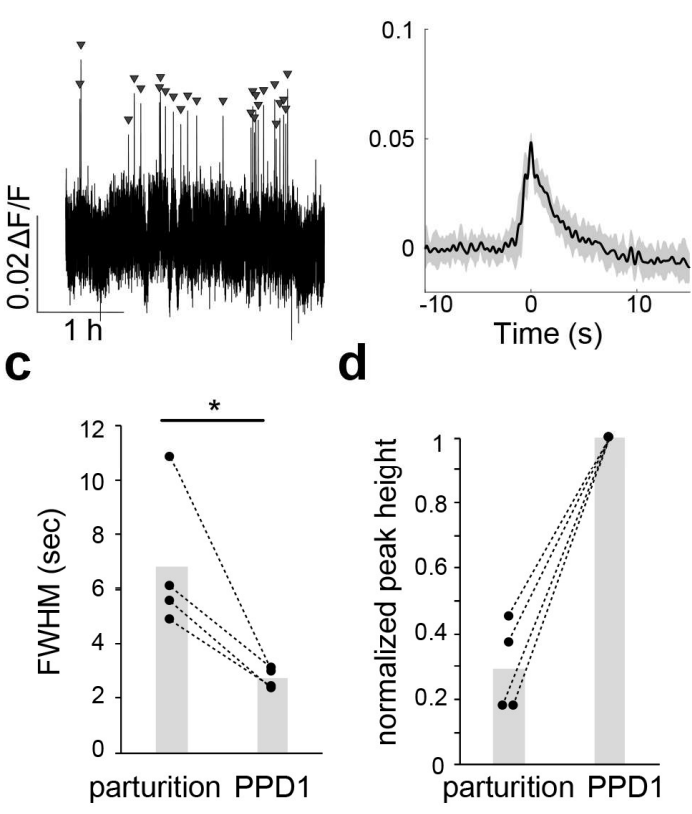

Supplementary Fig. 3 OT-PAs in the secundiparous mothers and quantitative comparison of the waveform between parturition and lactation, related to Fig. 2

a Timeline of the experiments. In most cases, we recorded $\mathrm{Ca}^{2+}$ dynamics of OT neurons at PPD1 and PPD12 following the first parturition (\#1). For a small number of animals, we performed a second round of imaging following the second parturition (\#2) after weaning and re-crossing. b Representative examples of 3-h traces of fiber photometry data obtained from PPD1 ${ }^{\# 2}$ and PPD $12^{\# 2}$ mothers during lactation. Averaged peri-event traces of individual OT-PAs are shown on the right, with shadows showing the SD. Of note, the traces shown in Fig. $2 \mathrm{f}$ and this panel originated from the same animal. c Quantification of averaged FWHM during parturition and lactation at PPD1 analyzed in the same female mice. ${ }^{*}, \mathrm{p}=0.047$ by two-sided paired $t$-test. $n=4$ animals. $\mathbf{d}$ The mean of the peak height during parturition normalized to that during lactation at PPD1 detected from each female mouse. $\mathrm{p}=0.070$ by two-sided paired $t$-test. $n=4$ animals. 
Fiber photometry of maternal oxytocin pulses by Yukinaga et al.

a

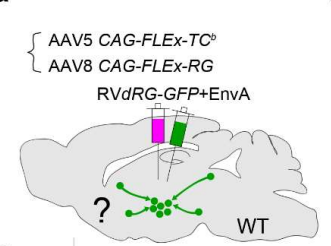

C

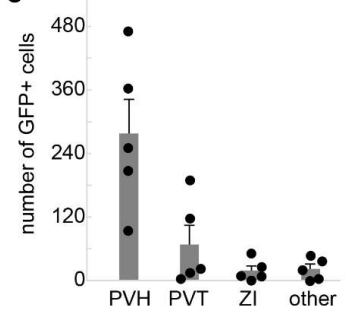

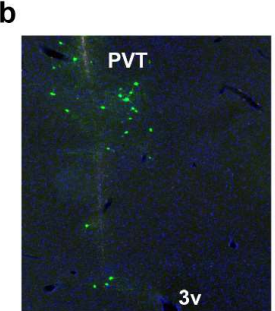

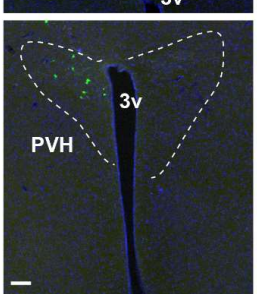

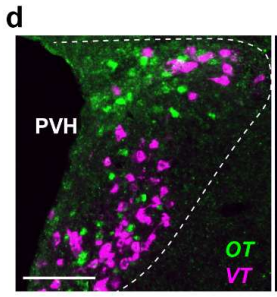

OT Flu probe

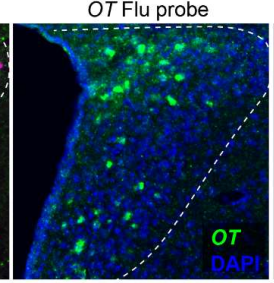

e

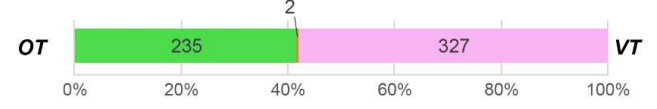

Supplementary Fig. 4 Nonspecific background labeling in the PVH and validation of ISH probes, related to Figs. 3 and 5

a Schematics of the experimental design. b Representative coronal sections showing Creindependent background labeling of rabies-GFP in the PVT (top) and PVH (bottom). c Quantification of GFP+ cells per animal. $n=5$. Error bars, SEM. ZI, Zona incerta. d Representative coronal section with $O T$ (Flu probe, green) and VT (Dig probe, magenta) ISH staining counterstained with DAPI (blue). e Quantification of OT- and VT-labeled cells from the PVH sections of two animals (pooled), showing that these two probes detected an almost mutually exclusive neural population. Scale bar, $100 \mu \mathrm{m}$. 
Fiber photometry of maternal oxytocin pulses by Yukinaga et al.

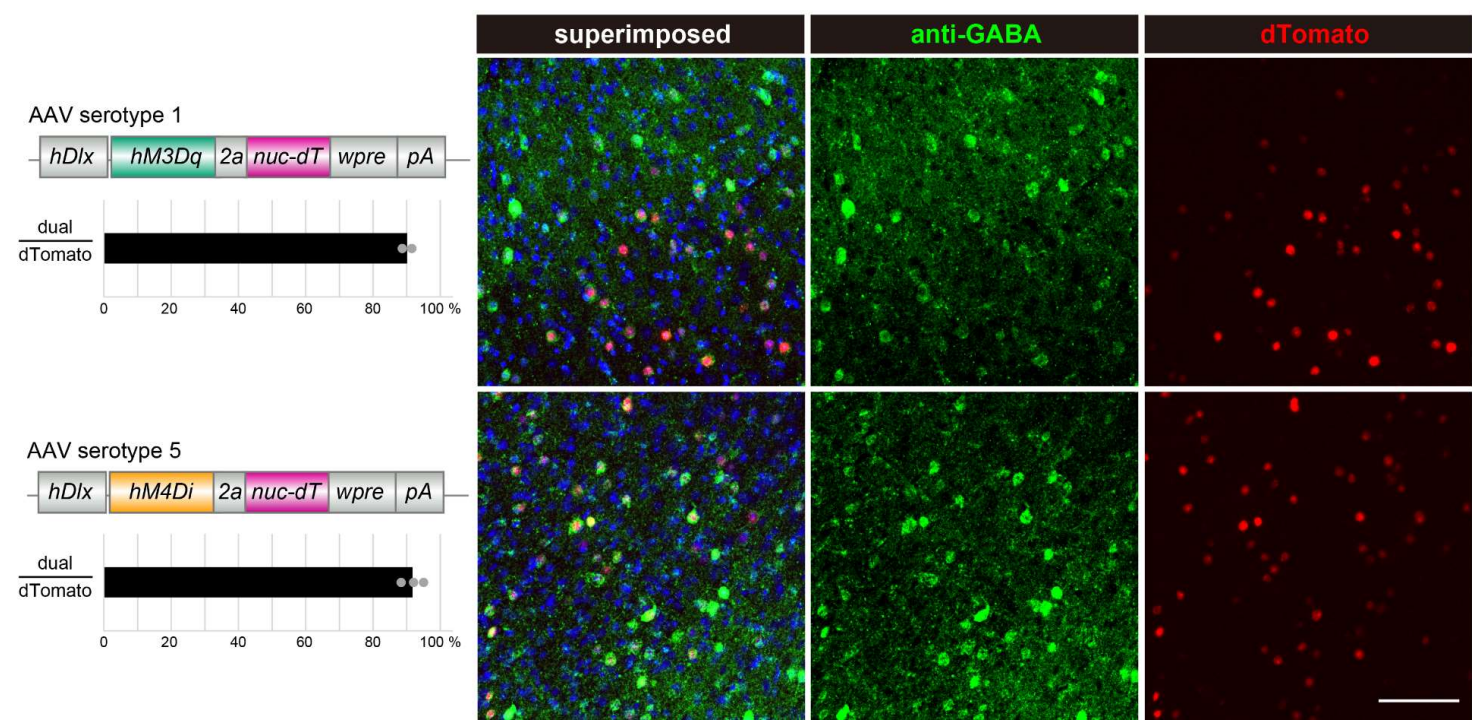

Supplementary Fig. 5 Validating inhibitory neuron-specific expression of hM3Dq and hM4Di transgenes driven by the $h D l x$ enhancer, related to Fig. 6

AAV1 $h D l x$-hM3Dq-2a-nuclear-dTomato or AAV5 hDlx-hM4Di-2a-nuclear-dTomato was injected into the motor cortex of wild-type mice, and dTomato-labeled cells were detected together with anti-GABA staining in the coronal brain sections. Left, schematics of the constructs and fraction of GABA and dTomato dual-labeled cells among dTomatopositive cells in the motor cortex. $n=2$ for hM3Dq vector and $n=3$ for hM4Di vector. Right, representative coronal brain sections of the motor cortex showing anti-GABA staining (green) and dTomato expression (red), counterstained with DAPI (blue). Scale bar, $100 \mu \mathrm{m}$.

\section{Supplementary movie: Dynamics of OT neural activities during parturition, related} to Fig. 2

The OT-Cre; Ail62 female mouse is about to give birth to her second pup in this parturition. Top, side (left), and bottom (right) view of the cage. Bottom, fiber photometry trace of GCaMP6s signals (the same sample shown in Fig. 1f). OT-PAs are indicated by arrowheads. The timing of abdominal contractions and pup delivery are represented by blue and red vertical dotted lines. Of note, abdominal contractions often occur 10-15 s after the OT-PAs. The video is shown at $4 \times$ real-time speed. 
Fiber photometry of maternal oxytocin pulses by Yukinaga et al.

\section{Methods}

Experimental protocols utilizing rabies virus followed Biosafety Level 2 (P2/P2A) procedures approved by the biosafety committee of the RIKEN Center for Biosystems Dynamics Research (BDR). All animal procedures followed animal care guidelines approved by the Institutional Animal Care and Use Committee of the RIKEN Kobe branch.

\section{Animals}

Animals were housed under a regular 12-h dark/light cycle with ad libitum access to food and water. Wild-type C57BL/6j mice were purchased from Japan SLC (Shizuoka, Japan) for histological and mating experiments. OT-Cre (Jax\# 024234) and Ail62 (TIT2L-GC6sICL-tTA2, Jax\#031562) were purchased from the Jackson Laboratory.

\section{Viral preparations}

The following AAV vectors were generated by UNC viral core using the corresponding plasmids as described in the original literature ${ }^{27}$. The titer of the AAV was estimated by quantitative PCR methods and shown as genome particles (gp) per milliliter.

AAV serotype 5 CAG-FLEx-TC $\left(9.3 \times 10^{12} \mathrm{gp} / \mathrm{ml}\right)$

AAV serotype $8 C A G-F L E x-R G\left(2.8 \times 10^{12} \mathrm{gp} / \mathrm{ml}\right)$

AAV serotype 2 CAG-FLEx-TC $C^{66 T}\left(1.0 \times 10^{12} \mathrm{gp} / \mathrm{ml}\right)$

AAV serotype 2 CAG-FLEx-RG $\left(1.3 \times 10^{12} \mathrm{gp} / \mathrm{ml}\right)$

The following AAV vectors were generated by the viral vector cores of Fukushima Medical University School of Medicine and Canadian Neurophotonics Platform by using the corresponding plasmids (Addgene \#83896 and \#83897) described in the original literature ${ }^{33}$.

AAV serotype 5 hDlx-hM3Dq-nuc-dTomato $\left(1.0 \times 10^{13} \mathrm{gp} / \mathrm{ml}\right)$

AAV serotype 1 hDlx-hM4Di-nuc-dTomato $\left(8.9 \times 10^{12} \mathrm{gp} / \mathrm{ml}\right)$

Rabies $d G-G F P+E n v A$ was prepared by using B7GG and BHK-EnvA cells (kindly gifted by Ed Callaway) according to the published protocol ${ }^{48}$. The EnvA-pseudotyped RV $d G$ $G F P+$ EnvA titer was estimated to be $1.0 \times 10^{9}$ infectious particles $/ \mathrm{ml}$ based on serial dilutions of the virus stock, followed by infection of the HEK293-TVA800 cell line.

\section{Stereotactic injection}

For targeting AAV or rabies virus into a certain brain region, stereotactic coordinates were 
Fiber photometry of maternal oxytocin pulses by Yukinaga et al.

first defined for each brain region based on the Allen Brain Atlas ${ }^{49}$. Mice were anesthetized with $65 \mathrm{mg} / \mathrm{kg}$ ketamine (Daiichi-Sankyo) and $13 \mathrm{mg} / \mathrm{kg}$ xylazine (SigmaAldrich) via intraperitoneal injection and head-fixed to the stereotactic equipment (Narishige). For rabies tracing experiments (Figs. 3, 4), $200 \mathrm{nl}$ of 1:1 mixture of AAV5 $C A G-F L E x-T C^{b}$ and AAV8 CAG-FLEx-RG was injected into the PVH at a speed of 50 nl/min using a UMP3 pump regulated by Micro-4 (World Precision Instruments). For local circuit mapping (Fig. 5), $200 \mathrm{nl}$ of 1:1 mixture of AAV2 CAG-FLEx-TC ${ }^{66 T}$ and AAV2 CAG-FLEx-RG was injected into the PVH, followed by $200 \mathrm{nl}$ of Rabies $d G$ $G F P+$ EnvA injection to the same coordinate 2 weeks later. For pharmaco-genetic experiments (Fig. 6), $200 \mathrm{nl}$ of AAV5 hDlx-hM4Di-2a-nuc-dTomato or AAV1 hDlx$h M 3 D q-2 a-n u c-d T o m a t o$ was injected into the septum/BST. For testing hDlx driving AAVs (Supplementary Fig. 5), $200 \mathrm{nl}$ of AAV5 hDlx-hM4Di-2a-nuc-dTomato or AAV1 $h D l x-h M 3 D q-2 a-n u c-d T o m a t o$ was injected into the primary motor cortex (M1). The following coordinates were used (Distance in millimeters from the Bregma for the anterior $[\mathrm{A}]-$ posterior $[\mathrm{P}]$ and lateral $[\mathrm{L}]$ positions, and from the brain surface for the ventral [V] direction)]: M1, A 1.5, L 1.2, V 0.45; septum/BST, A 0.2, L 0.6, V 3.7; and $\mathrm{PVH}, \mathrm{A}-0.75 \mathrm{~L} 0.2, \mathrm{~V} 4.5$. After viral injection, the incision was sutured, and the animal was warmed using a heating pad to facilitate recovery from anesthesia. The animal was then returned to the home cage.

\section{Fiber photometry}

For fiber photometry recording, Ai162/+; OT-Cre/+ double heterozygous female mice were used. A $400-\mu \mathrm{m}$ core, 0.5 NA optical fiber (Thorlabs, cat\#FP400URT) was implanted immediately above the PVH. After the surgery, animals were crossed with stud males and housed in the home cage until recording. We performed $\mathrm{Ca}^{2+}$ imaging by delivering excitation lights (465-nm modulated at $309.944 \mathrm{~Hz}$ and $405-\mathrm{nm}$ modulated at $208.616 \mathrm{~Hz}$ ) and collect emitted fluorescence by using the integrated Fluorescence Mini Cube (Doric, iFMC4_AE(405)_E(460-490)_F(500-550)_S). Light collection, filtering, and demodulation were performed using Doric photometry setup and Doric Neuroscience Studio Software (Doric Lenses, Inc.). The 405-nm signal was recorded as a background (non-calcium-dependent), and the 465-nm signal reported calcium-dependent GCaMP6s excitation/emission. The power output at the tip of the fiber was about $5 \mu \mathrm{W}$. The signals were initially acquired at $12 \mathrm{kHz}$ and then decimated to $120 \mathrm{~Hz}$ for recording to disk. As the 405-nm signals were flat during OT-PAs in parturition (Fig. 1f) or lactation (not shown), we did not apply further background subtraction methods. We used a 2-Hz lowpass filter before the analysis of OT-PAs. 
Fiber photometry of maternal oxytocin pulses by Yukinaga et al.

For the analyses, we used a homemade MATLAB code. Briefly, the $\Delta \mathrm{F} / \mathrm{F}$ was calculated by $100 \times\left(\mathrm{F}_{t}-\mathrm{F}_{0}\right) / \mathrm{F}_{0}$, where $\mathrm{F}_{t}$ was the recorded signal at time $=t$ and $\mathrm{F}_{0}$ was the average of signals in the whole recording period. Because the height of OT-PAs in each mother varied considerably, as the optical fiber location relative to the PVH was variable, to identify the OT-PAs reliably, we first selected several visually obvious peaks to estimate the peak height of that animal. OT-PAs were then automatically detected by using the findpeaks function in MATLAB, with the peak threshold of half of the estimated peak height, and the FWHM threshold over $4 \mathrm{~s}$. To show the peri-event traces of OT-PAs (Figs. 1g, 2b, f, h, i, 6c, d, and Supplementary Fig. 3b), we extracted the $\Delta F / F$ data from $-10 \mathrm{~s}$ to $+15 \mathrm{~s}$ around the peak (the local maximum point of $\Delta \mathrm{F} / \mathrm{F}$ ). We then aligned the multiple pulses by adjusting the median fluorescence of the -8 to $-3 \mathrm{~s}$ baseline period to zero.

Videos from a side infrared camera (DMK33UX273; The Imaging Source) were synchronized with fiber photometry acquisition. The camera from the bottom (QWatch; IODATA) was matched with the side camera using the mouse motion and light conditions. For the analysis of parturition, based on the bottom view of the video record, the delivery timing was defined as the moment when the entire pup was outside the vagina. We extracted the $\Delta \mathrm{F} / \mathrm{F}$ data from $-10 \mathrm{~min}$ to $+5 \mathrm{~min}$ around each delivery and analyzed only the peaks detected before delivery because those after delivery might be included in the next delivery event. For the heat map representation (Fig. 1h), $\Delta \mathrm{F} / \mathrm{F}$ was normalized to the maximum value within that animal in a $-10 \mathrm{~min}$ to $+5 \mathrm{~min}$ period of the analysis. To compare peak height (Fig. 2g, j, Supplementary Fig. 3d), we calculated the averaged peak height of one condition normalized to the averaged peak height of another condition of the same animal to obtain the normalized $\Delta \mathrm{F} / \mathrm{F}$. For the analysis of OT-PAs during lactation, PPD1 was defined as 1 day after the day of parturition. In the cross-fostering experiments (Fig. 2h-j), we used the postnatal day 0-1 pup as 'young' and the postnatal day $10-16$ pup as 'elder'.

\section{Pharmacogenetics}

Neural activation or inactivation experiments were performed at least 2 weeks after the injection of AAV driving hM3Dq or hM4Di. Then, $0.3 \mathrm{ml}$ of $400 \mu \mathrm{g} / \mathrm{ml} \mathrm{CNO}$ (SigmaAldrich cat\#C0832) dissolved in saline or $0.3 \mathrm{ml}$ of saline was intraperitoneally injected into the animal during the fiber photometry imaging sessions.

\section{Histology and histochemistry}

For the quantitative analysis of the trans-synaptic tracing samples and the histochemical 
Fiber photometry of maternal oxytocin pulses by Yukinaga et al.

analyses, the experimental mice were anesthetized with a lethal amount of sodium pentobarbital, sacrificed, and perfused with phosphate-buffered saline (PBS) followed by $4 \%$ paraformaldehyde (PFA) in PBS. Brain tissues were post-fixed with 4\% PFA in PBS overnight at $4{ }^{\circ} \mathrm{C}$, cryoprotected with $30 \%$ sucrose solution in PBS at $4{ }^{\circ} \mathrm{C}$ for $24-48 \mathrm{~h}$, and embedded in the O.C.T. compound (Tissue-Tek, cat\#4583). We collected 30- $\mu \mathrm{m}$ coronal sections of the whole brain using Cryostat (model \#CM1860; Leica) and placed them on MAS-coated glass slides (Matsunami). Unless otherwise noted, every third (Figs. 3, 4, and 6) or fifth (Figs. 1 and 5, Supplementary Figs. 4 and 5) coronal brain section was analyzed for quantification, and compensated data $(\times 3$ or $\times 5)$ were represented.

To map the long-distance input to OT neurons (Fig. 3), every third of the coronal brain section was imaged using a slide scanner (Zeiss Axio Scan.Z1) with a $20 \times$ objective lens (NA 0.8). Image processing was performed semi-automatically using ImageJ macro (National Institutes of Health, Bethesda, MD). Briefly, regions of interest (ROIs) were manually set for each brain region by using the DAPI channel (showing brain structures but not GFP+ cells) based on the Allen Brain Atlas ${ }^{49}$ by annotators who were blinded to the experimental conditions. For each ROI, the raw fluorescent image for GFP or mCherry was processed using open and median filters. The labeled cells were detected by using the "Threshold" and "Analyze Particles" commands. The starter cells were defined by those detected as cells using both GFP and mCherry channels. In Fig. 3f, the $d^{\prime}$ value was calculated as $\left(\mu_{\text {mother }}-\mu_{\text {nonpregnancy }}\right)$ divided by $\left(\sigma_{\text {mother }}+\sigma_{\text {nonpregnancy }}\right)$, where $\mu$ and $\sigma$ denote the average and standard deviation, respectively. A d' value larger than 0 means that OT neurons in lactating mothers receive more input.

Thirty-micrometer coronal sections containing the target brain region were subjected to ISH (Figs. 4, 5, Supplementary Fig. 4), as described previously ${ }^{50}$. To generate cRNA probes, DNA templates were amplified by PCR from the C57BL/6j mouse genome or whole-brain cDNA (Genostaff, cat\#MD-01). T3 RNA polymerase recognition site (5'AATTAACCCTCACTAAAGGG) was added to the 3' end of the reverse primers. Primer sets and sequences of probe targets are as follows (the first one, forward primer, the second one, reverse primer):

OT 5'-TGGCTTACTGGCTCTGACCT; 5'-AGGAAGCGCGCTAAAGGTAT VT 5'-CGCTCACAGAGCTCTTCCTT; 5'-GACACCAGGGTGCAGTTTTT vGluT2-1 5'-TAGCTTCCTCTGTCCGTGGT; 5'-GGGCCAAAATCCTTTGTTTT vGluT2-2 5'-CCACCAAATCTTACGGTGCT; 5'-GGAGCATACCCCTCCCTTTA vGluT2-3 5'-CTCCCCCATTCACTACCTGA; 5'-GGTCAGGAGTGGTTTGCATT vGAT1-1 5'-CCTGGTCTGGACAGCATCTC; 5'-GCTATGGCCACATACGAGTC vGAT1-2 5'-GTCAATGTGGCGCAGATCAT; 5'-CCTAGTCCTCTGCGTTGGTT 
bioRxiv preprint doi: https://doi.org/10.1101/2021.07.26.453888; this version posted July 26, 2021. The copyright holder for this preprint (which was not certified by peer review) is the author/funder, who has granted bioRxiv a license to display the preprint in perpetuity. It is made available under aCC-BY-NC-ND 4.0 International license.

Fiber photometry of maternal oxytocin pulses by Yukinaga et al.

DNA templates (500-1000 ng) amplified by PCR were subjected to in vitro transcription with DIG (cat\#11277073910) or Flu (cat\#11685619910)-RNA labeling mix and T3 RNA polymerase (cat\#11031163001) according to the manufacturer's instructions (Roche). When possible, two or three independent RNA probes for the same gene were mixed to increase the signal/noise ratio.

For single-color ISH combined with anti-GFP staining, after hybridization and washing, sections were incubated with horseradish peroxidase (HRP)-conjugated antiDig (Roche Applied Science cat\#11207733910, 1:500) and anti-GFP (Aves Labs cat\#GFP-1020, 1:500) antibodies overnight. Signals were amplified by TSA-plus Cyanine 3 (AKOYA Bioscience, NEL744001KT, 1:70 in 1× plus amplification diluent) for $25 \mathrm{~min}$, followed by washing, and then GFP-positive cells were visualized by antichicken Alexa Fluor 488 (Invitrogen, cat\#A11039, 1:250). PBS containing 50 ng/ml 4',6diamidino-2-phenylindole dihydrochloride (DAPI; Sigma-Aldrich, cat\#D8417) was used for counter nuclear staining.

For dual-color ISH, HRP-conjugated anti-flu antibody (AKOYA Bioscience NEF710001EA, 1:250 in blocking buffer) was used to detect the Flu-labeled RNA probe by TSA-plus biotin (AKOYA Bioscience, NEL749A001KT, 1:70 in 1× plus amplification diluent), which was then visualized with streptavidin-Alexa Fluor 488 (Life Technologies, 1:250). After inactivation of HRP with $2 \%$ sodium azide in PBS for 15 min, HRPconjugated anti-Dig (Roche Applied Science cat\#11207733910, 1:500) and TSA-plus Cyanine 3 (1:70 in $1 \times$ plus amplification diluent) were used to detect the Dig-labeled cRNA probe. Sections were mounted with cover glass using Fluoromount (Diagnostic BioSystems cat\#K024).

For immunohistochemistry, sections were washed three times with PBS containing $0.3 \%$ Tween-20 (PBST) for $10 \mathrm{~min}$ and treated with 5\% normal donkey serum (NDS; Southern Biotech, cat\#0030-01) in PBST for $1 \mathrm{~h}$ at room temperature for blocking. The following primary antibodies were used in this study: rat anti-mCherry (Life Technologies cat\#M11217, 1:500-1:1000), chicken anti-GFP (Aves Labs cat\#GFP-1020, 1:500), rabbit anti-GABA (Merck A2052, 1:2000), and rabbit anti-OT (Immunostar cat\#20068, 1:1000). These antibodies were diluted into 5\% NDS in PBST for $3 \mathrm{~h}$ at room temperature or overnight at $4{ }^{\circ} \mathrm{C}$. Signal-positive cells were detected by the following secondary antibodies: anti-rat Cy3 (Millipore, cat\#AP189C, 1:250), anti-chicken Alexa Fluor 488 (Invitrogen cat\#A11039, 1:250), anti-rabbit Alexa Fluor 488 (Invitrogen, cat\#A32790, 1:250), and anti-rabbit Alexa Fluor 555 (Invitrogen cat\#32794, 1:250) diluted into PBST for $2 \mathrm{~h}$ at room temperature or overnight at $4{ }^{\circ} \mathrm{C}$. Sections were washed once with PBST for $10 \mathrm{~min}$, treated with PBS containing DAPI for $20 \mathrm{~min}$, rinsed with 
bioRxiv preprint doi: https://doi.org/10.1101/2021.07.26.453888; this version posted July 26, 2021. The copyright holder for this preprint (which was not certified by peer review) is the author/funder, who has granted bioRxiv a license to display the preprint in perpetuity. It is made available under aCC-BY-NC-ND 4.0 International license.

Fiber photometry of maternal oxytocin pulses by Yukinaga et al.

PBS, and mounted with cover glass using Fluoromount (Diagnostic BioSystems cat\#K024).

Sections were imaged using an Olympus BX53 microscope with a $4 \times($ NA 0.16$)$ or 10× (NA 0.4) objective lens equipped with a cooled CCD camera (DP80; Olympus) or Zeiss Axio Scan.Z1 with a 20× (NA 0.8) objective lens. Images were processed in ImageJ and Photoshop CC (Adobe).

\section{Data availability}

All relevant data and codes are available from the authors upon reasonable request. 
Fiber photometry of maternal oxytocin pulses by Yukinaga et al.

\section{References}

1 Armstrong, W. E. Central Nervous System Control of Oxytocin Secretion during Lactation. Physiology of Reproduction 5th edition, 527-560 (2015).

2 Brunton, P. J. R., J. A. Maternal Brain Adaptations in Pregnancy. Physiology of Reproduction 5th edition, 1957-2026 (2015).

3 Froemke, R. C. C., I. Oxytocin and Brain Plasticity. Principles of Gender-Specific Medicine, 161-182 (2017).

4 Nishimori, K. et al. Oxytocin is required for nursing but is not essential for parturition or reproductive behavior. Proc Natl Acad Sci U S A 93, 11699-11704, doi:10.1073/pnas.93.21.11699 (1996).

$5 \quad$ Young, W. S., 3rd et al. Deficiency in mouse oxytocin prevents milk ejection, but not fertility or parturition. $J$ Neuroendocrinol 8, 847-853, doi:10.1046/j.13652826.1996.05266.x (1996).

6 Takayanagi, Y. et al. Pervasive social deficits, but normal parturition, in oxytocin receptor-deficient mice. Proc Natl Acad Sci U S A 102, 16096-16101, doi:10.1073/pnas.0505312102 (2005).

7 Douglas, A. J., Leng, G. \& Russell, J. A. The importance of oxytocin mechanisms in the control of mouse parturition. Reproduction 123, 543-552, doi:10.1530/rep.0.1230543 (2002).

8 Bell, A. F., Erickson, E. N. \& Carter, C. S. Beyond labor: the role of natural and synthetic oxytocin in the transition to motherhood. J Midwifery Womens Health 59, 35-42: quiz 108, doi:10.1111/jmwh.12101 (2014).

9 Wakerley, J. B. \& Lincoln, D. W. The milk-ejection reflex of the rat: a 20- to 40-fold acceleration in the firing of paraventricular neurones during oxytocin release. $J$ Endocrinol 57, 477-493, doi:10.1677/joe.0.0570477 (1973).

10 Lincoln, D. W. \& Wakerley, J. B. Factors governing the periodic activation of supraoptic and paraventricular neurosecretory cells during suckling in the rat. $J$ Physiol 250, 443-461, doi:10.1113/jphysiol.1975.sp011064 (1975).

11 Sutherland, R. C., Juss, T. S. \& Wakerley, J. B. Prolonged electrical stimulation of the nipples evokes intermittent milk ejection in the anaesthetised lactating rat. Exp Brain Res 66, 29-34, doi:10.1007/BF00236198 (1987).

12 Belin, V. \& Moos, F. Paired recordings from supraoptic and paraventricular oxytocin cells in suckled rats: recruitment and synchronization. $J$ Physiol 377, 369-390, doi:10.1113/jphysiol.1986.sp016192 (1986).

13 O'Byrne, K. T., Ring, J. P. \& Summerlee, A. J. Plasma oxytocin and oxytocin neurone activity during delivery in rabbits. $J$ Physiol 370, 501-513, 
Fiber photometry of maternal oxytocin pulses by Yukinaga et al.

doi:10.1113/jphysiol.1986.sp015947 (1986).

14 Paisley, A. C. \& Summerlee, A. J. Activity of putative oxytocin neurones during reflex milk ejection in conscious rabbits. $J$ Physiol 347, 465-478, doi:10.1113/jphysiol.1984.sp015076 (1984).

15 Summerlee, A. J. \& Lincoln, D. W. Electrophysiological recordings from oxytocinergic neurones during suckling in the unanaesthetized lactating rat. $J$ Endocrinol 90, 255265, doi:10.1677/joe.0.0900255 (1981).

16 Lewis, E. M. et al. Parallel Social Information Processing Circuits Are Differentially Impacted in Autism. Neuron 108, 659-675 e656, doi:10.1016/j.neuron.2020.10.002 (2020).

17 Zhang, B. et al. Reconstruction of the Hypothalamo-Neurohypophysial System and Functional Dissection of Magnocellular Oxytocin Neurons in the Brain. Neuron 109, 331-346 e337, doi:10.1016/j.neuron.2020.10.032 (2021).

18 Luo, L., Callaway, E. M. \& Svoboda, K. Genetic Dissection of Neural Circuits: A Decade of Progress. Neuron 98, 256-281, doi:10.1016/j.neuron.2018.03.040 (2018).

19 Hung, L. W. et al. Gating of social reward by oxytocin in the ventral tegmental area. Science 357, 1406-1411, doi:10.1126/science.aan4994 (2017).

20 Tang, Y. et al. Social touch promotes interfemale communication via activation of parvocellular oxytocin neurons. Nat Neurosci 23, 1125-1137, doi:10.1038/s41593-0200674-y (2020).

21 Carcea, I. et al. Oxytocin Neurons Enable Social Transmission of Maternal Behavior. bioRxiv, 845495, doi:10.1101/845495 (2020).

22 Valtcheva, S. et al. Neural circuitry for maternal oxytocin release induced by infant cries. bioRxiv, 2021.2003.2025.436883, doi:10.1101/2021.03.25.436883 (2021).

23 Dubois-Dauphin, M., Armstrong, W. E., Tribollet, E. \& Dreifuss, J. J. Somatosensory systems and the milk-ejection reflex in the rat. II. The effects of lesions in the ventroposterior thalamic complex, dorsal columns and lateral cervical nucleusdorsolateral funiculus. Neuroscience 15, 1131-1140, doi:10.1016/0306-4522(85)90257$\mathrm{x}(1985)$.

24 Honda, K. \& Higuchi, T. Effects of unilateral electrolytic lesion of the dorsomedial nucleus of the hypothalamus on milk-ejection reflex in the rat. J Reprod Dev 56, 98102, doi:10.1262/jrd.09-090e (2010).

25 Lebrun, C. J., Poulain, D. A. \& Theodosis, D. T. The role of the septum in the control of the milk ejection reflex in the rat: effects of lesions and electrical stimulation. $J$ Physiol 339, 17-31, doi:10.1113/jphysiol.1983.sp014699 (1983).

26 Gunaydin, L. A. et al. Natural neural projection dynamics underlying social behavior. 
Fiber photometry of maternal oxytocin pulses by Yukinaga et al.

Cell 157, 1535-1551, doi:10.1016/j.cell.2014.05.017 (2014).

27 Miyamichi, K. et al. Dissecting local circuits: parvalbumin interneurons underlie broad feedback control of olfactory bulb output. Neuron 80, 1232-1245, doi:10.1016/j.neuron.2013.08.027 (2013).

28 Wu, Z. et al. An obligate role of oxytocin neurons in diet induced energy expenditure. PLoS One 7, e45167, doi:10.1371/journal.pone.0045167 (2012).

29 Daigle, T. L. et al. A Suite of Transgenic Driver and Reporter Mouse Lines with Enhanced Brain-Cell-Type Targeting and Functionality. Cell 174, 465-480 e422, doi:10.1016/j.cell.2018.06.035 (2018).

30 Miyamichi, K. et al. Cortical representations of olfactory input by trans-synaptic tracing. Nature 472, 191-196, doi:10.1038/nature09714 (2011).

31 Knobloch, H. S. et al. Evoked axonal oxytocin release in the central amygdala attenuates fear response. Neuron 73, 553-566, doi:10.1016/j.neuron.2011.11.030 (2012).

32 Theofanopoulou, C., Gedman, G., Cahill, J. A., Boeckx, C. \& Jarvis, E. D. Universal nomenclature for oxytocin-vasotocin ligand and receptor families. Nature 592, 747755, doi:10.1038/s41586-020-03040-7 (2021).

33 Dimidschstein, J. et al. A viral strategy for targeting and manipulating interneurons across vertebrate species. Nat Neurosci 19, 1743-1749, doi:10.1038/nn.4430 (2016).

34 Armbruster, B. N., Li, X., Pausch, M. H., Herlitze, S. \& Roth, B. L. Evolving the lock to fit the key to create a family of $\mathrm{G}$ protein-coupled receptors potently activated by an inert ligand. Proc Natl Acad Sci $U$ S A 104, 5163-5168, doi:10.1073/pnas.0700293104 (2007).

35 Marlin, B. J., Mitre, M., D'Amour J, A., Chao, M. V. \& Froemke, R. C. Oxytocin enables maternal behaviour by balancing cortical inhibition. Nature 520, 499-504, doi:10.1038/nature14402 (2015).

36 Choe, H. K. et al. Oxytocin Mediates Entrainment of Sensory Stimuli to Social Cues of Opposing Valence. Neuron 87, 152-163, doi:10.1016/j.neuron.2015.06.022 (2015).

37 Yao, S., Bergan, J., Lanjuin, A. \& Dulac, C. Oxytocin signaling in the medial amygdala is required for sex discrimination of social cues. Elife 6, doi:10.7554/eLife.31373 (2017).

38 Nakajima, M., Gorlich, A. \& Heintz, N. Oxytocin modulates female sociosexual behavior through a specific class of prefrontal cortical interneurons. Cell 159, 295305, doi:10.1016/j.cell.2014.09.020 (2014).

39 Li, K., Nakajima, M., Ibanez-Tallon, I. \& Heintz, N. A Cortical Circuit for Sexually Dimorphic Oxytocin-Dependent Anxiety Behaviors. Cell 167, 60-72 e11, 
Fiber photometry of maternal oxytocin pulses by Yukinaga et al.

doi:10.1016/j.cell.2016.08.067 (2016).

40 Summerlee, A. J. Extracellular recordings from oxytocin neurones during the expulsive phase of birth in unanaesthetized rats. $J$ Physiol 321, 1-9, doi:10.1113/jphysiol.1981.sp013967 (1981).

41 Ghosh, K. K. et al. Miniaturized integration of a fluorescence microscope. Nat Methods 8, 871-878, doi:10.1038/nmeth.1694 (2011).

42 Son, S. et al. Wiring diagram of the oxytocin system in the mouse brain. bioRxiv, 2020.2010.2001.320978, doi:10.1101/2020.10.01.320978 (2020).

43 Theodosis, D. T., Piet, R., Poulain, D. A. \& Oliet, S. H. Neuronal, glial and synaptic remodeling in the adult hypothalamus: functional consequences and role of cell surface and extracellular matrix adhesion molecules. Neurochem Int 45, 491-501, doi:10.1016/j.neuint.2003.11.003 (2004).

44 Moos, F. et al. Oxytocin in the bed nucleus of the stria terminalis and lateral septum facilitates bursting of hypothalamic oxytocin neurons in suckled rats. $J$ Neuroendocrinol 3, 163-171, doi:10.1111/j.1365-2826.1991.tb00259.x (1991).

45 Dulac, C. \& Wagner, S. Genetic analysis of brain circuits underlying pheromone signaling. Annu Rev Genet 40, 449-467, doi:10.1146/annurev.genet.39.073003.093937 (2006).

46 Kim, S. R. \& Kim, S. Y. Functional Dissection of Glutamatergic and GABAergic Neurons in the Bed Nucleus of the Stria Terminalis. Mol Cells 44, 63-67, doi:10.14348/molcells.2021.0006 (2021).

47 Dewey, K. G. Maternal and fetal stress are associated with impaired lactogenesis in humans. J Nutr 131, 3012S-3015S, doi:10.1093/jn/131.11.3012S (2001).

48 Osakada, F. \& Callaway, E. M. Design and generation of recombinant rabies virus vectors. Nat Protoc 8, 1583-1601, doi:10.1038/nprot.2013.094 (2013).

49 Lein, E. S. et al. Genome-wide atlas of gene expression in the adult mouse brain. Nature 445, 168-176, doi:10.1038/nature05453 (2007).

50 Ishii, K. K. et al. A Labeled-Line Neural Circuit for Pheromone-Mediated Sexual Behaviors in Mice. Neuron 95, 123-137 e128, doi:10.1016/j.neuron.2017.05.038 (2017). 
bioRxiv preprint doi: https://doi.org/10.1101/2021.07.26.453888; this version posted July 26, 2021. The copyright holder for this preprint (which was not certified by peer review) is the author/funder, who has granted bioRxiv a license to display the preprint in perpetuity. It is made available under aCC-BY-NC-ND 4.0 International license.

Fiber photometry of maternal oxytocin pulses by Yukinaga et al.

\section{Authors' contributions}

H.Y. and K.M. designed the study. H.Y. performed the fiber photometry recordings, retrograde trans-synaptic tracing, and pharmaco-genetic manipulation experiments with technical support from M.H., K.T., and C.H.L. S.K. and K.K. provided the AAV Dlx$D R E A D D$ viruses. H.Y. and K.M. wrote the manuscript with assistance from all coauthors.

\section{Acknowledgments}

We wish to thank Kengo Inada, Teppei Goto, and Ken Murata for technical advice on fiber photometry, E. Callaway for sharing the B7GG, BHK-EnvA, and HEK293-TVA800 cell lines, and all the laboratory members for their help and critical reading of the manuscript. This work was supported by Kakenhi 17K14948 and 20K15907 to H.Y., AMED Brain/MINDS program 20dm0207052h0004 to K.K., and JST PREST program JPMJPR1789, Kakenhi 18H02548 and 20K20589, a Takeda Science Foundation Research Grant, and The Uehara Memorial Foundation Research Grant to K.M. 